\title{
Neuroprotective effect of local hypothermia in a computer-controlled compression model in minipig: Correlation of tissue sparing along the rostro-caudal axis with neurological outcome
}

\author{
STEFANIA GEDROVA $^{1}$, JAN GALIK $^{1}$, MARTIN MARSALA ${ }^{1,2}$, MONIKA ZAVODSKA $^{1}$, \\ JAROSLAV PAVEL ${ }^{1}$, IGOR SULLA ${ }^{1,3}$, MIROSLAV GAJDOS $^{4}$, IMRICH LUKAC ${ }^{4}$, JOZEF KAFKA ${ }^{4}$, \\ VALENT LEDECKY ${ }^{5}$, IGOR SULLA JR. ${ }^{5}$, MARTINA KARASOVA ${ }^{5}$, PETER REICHEL $^{5}$, \\ ALEXANDRA TRBOLOVA ${ }^{5}$, IGOR CAPIK ${ }^{5}$, VIKTORIA LUKACOVA ${ }^{6}$, KATARINA BIMBOVA ${ }^{1}$, \\ MARIA BACOVA $^{1}$, ANDREA STROPKOVSKA ${ }^{1}$ and NADEZDA LUKACOVA ${ }^{1}$ \\ ${ }^{1}$ Institute of Neurobiology, Slovak Academy of Sciences, 04001 Kosice, Slovak Republic; \\ ${ }^{2}$ Neuroregeneration Laboratory, Department of Anesthesiology, University of California-San Diego, La Jolla, \\ CA 92037, USA; ${ }^{3}$ Hospital of Slovak Railways, 04001 Kosice; ${ }^{4}$ Department of Neurosurgery, Faculty of Medicine, \\ University of Pavol Jozef Safarik, 04001 Kosice; ${ }^{5}$ University of Veterinary Medicine and Pharmacy, 04181 Kosice; \\ ${ }^{6}$ Faculty of Economics, Technical University of Kosice, 04001 Kosice, Slovak Republic
}

Received June 7, 2017; Accepted September 20, 2017

DOI: $10.3892 /$ etm.2017.5432

\begin{abstract}
This study investigated the neuroprotective efficacy of local hypothermia in a minipig model of spinal cord injury (SCI) induced by a computer-controlled impactor device. The tissue integrity observed at the injury epicenter, and up to $3 \mathrm{~cm}$ cranially and caudally from the lesion site correlated with motor function. A computer-controlled device produced contusion lesions at L3 level with two different degrees of tissue sparing, depending upon pre-set impact parameters $(8 \mathrm{~N}$ and $15 \mathrm{~N}$-force impact). Hypothermia with cold $\left(4^{\circ} \mathrm{C}\right)$ saline or Dulbecco's modified Eagle's medium (DMEM)/F12 culture medium was applied $30 \mathrm{~min}$ after SCI (for $5 \mathrm{~h}$ ) via a perfusion chamber (flow $2 \mathrm{ml} / \mathrm{min}$ ). After saline hypothermia, the $8 \mathrm{~N}-\mathrm{SCI}$ group achieved faster recovery of hind limb function and the ability to walk from one to three steps at nine weeks in comparison with non-treated animals. Such improvements were not observed in saline-treated animals subjected to more severe $15 \mathrm{~N}-\mathrm{SCI}$ or in the group treated with DMEM/F12 medium. It was demonstrated that the tissue preservation in the cranial and caudal segments immediately adjacent to the
\end{abstract}

Correspondence to: Dr Jan Galik or Dr Nadezda Lukacova, Institute of Neurobiology, Slovak Academy of Sciences, Soltesovej 4-6, 04001 Kosice, Slovak Republic

E-mail: lukacova@saske.sk

E-mail: galik@saske.sk

Key words: spinal cord, local hypothermia, minipigs, 3D perfusion chamber, hindlimb function, tissue integrity, neurofilaments lesion, and neurofilament protection in the lateral columns may be essential for modulation of the key spinal microcircuits leading to a functional outcome. Tissue sparing observed only in the caudal sections, even though significant, was not sufficient for functional improvement in the $15 \mathrm{~N}-\mathrm{SCI}$ model.

\section{Introduction}

Currently there is robust experimental and some clinical evidence that hypothermia (local or systemic) may target multiple pathological mechanisms caused by SCI trauma. In principle, hypothermia reduces metabolic consumption and energy demands, having beneficial effects on cytoplasmic ATP stores and the maintenance of normal transmembrane ion and neurotransmitter gradients (1). Previous investigations have shown that the degree of ATP preservation depends on the hypothermic level as well as the severity of the insult. Erecinska et al (2) reported that hypothermia lowered the cerebral metabolic rate for oxygen and glucose two-fold to four-fold per $10^{\circ} \mathrm{C}$ reduction in temperature, in addition to reducing cerebral blood flow. Hypothermia can also provide benefits through its ability to preserve the blood-spinal cord barrier (BSCB), ameliorate local edema, suppress axonal swelling and development of gliosis in the peri-injury zones $(3,4)$; also to reduce oxidative stress, apoptosis and inflammation (5-8). Spinal cord injury studies in animals treated with mild systemic hypothermia for $30 \mathrm{~min}$ to $4 \mathrm{~h}$ have provided positive results for locomotor function and neuronal tissue maintenance. The protective influence of hypothermia has also been reported following its local application to the CNS. The advantage of local cooling using low temperature applied directly to the epicenter of injury obviates many of the 
risks of systemic hypothermia, such as arterial hypertension, bradycardia or pneumonia $(9,10)$. Better outcomes have been achieved when modest cooling was started as soon as possible after trauma $(4,5,8,11,12)$.

The development of a treatment strategy for acute spinal cord injury requires reliable and easily reproducible animal models allowing further characterization and better understanding of secondary pathophysiological mechanisms which could represent targets for therapeutic interventions. Preclinical and clinical trials have demonstrated that no treatment successful in rodents has been effective in humans so far. With regard to the similarity of their morphology and anatomical structures with humans, minipigs have already been selected as a useful SCI animal model for preclinical therapeutic trials $(13,14)$.

In the current study we used a computer-controlled chronic compression model in Göttingen-Minesota-Liběchov minipigs (15) to test local spinal cord hypothermia treatment with saline or oxygenated culture medium $\left(4^{\circ} \mathrm{C}\right)$ administered to the epicenter of injury beginning $30 \mathrm{~min}$ after traumatic impact. Because previous experimental and human clinical studies have shown effective transdural diffusion of epidurally administered analgesics, anesthetics and antibiotics (16-18), we anticipated that transdural diffusion of the saline or oxygenated culture medium at the lesion site without opening the spinal dural sac could lead to a positive treatment effect by maintaining local cellular homeostasis. Local hypothermia carried out through a special spinal perfusion chamber, prepared using 3D scanner technology, provided outright $5 \mathrm{~h}$-long perfusion of the lesion site (L3) with cold solutions $\left(4^{\circ} \mathrm{C}\right)$ administered by means of a peristaltic pump (flow $2 \mathrm{ml} / \mathrm{min}$ ), and allowed stable body temperature to be maintained during the whole perfusion period. The temperature of solutions in the perfusion chamber just above the lesion site was maintained at $19^{\circ} \mathrm{C}$.

The study provides evidence of considerable loss of neurofilament immunoreactivity (NF-IR), and reduced tissue degradation seen at the lesion site and away from the lesion epicenter ( $3 \mathrm{~cm}$ caudally as well as cranially) nine weeks after SCI ( $8 \mathrm{~N}$ and $15 \mathrm{~N}$ force), and it supports the beneficial effect of hypothermia with saline to promote the number of neurofilaments and gray and white matter preservation, and although without statistical significance, also functional improvement.

\section{Materials and methods}

Animals. Thirty adult female minipigs (Gottingen-MinnesotaLiběchov crossbred strains; 5-8 months of age, weighing 25-35 kg) were used in the experiment. The minipigs were delivered from Liběchov farm 3-4 months after their birth. They were group housed in connected pens and were maintained in standard conditions with ad libitum access to food and water. Female minipigs were used in order to avoid the development of penile prolapse that can be a serious complication after SCI performed at lumbar level. No estrus was observed in any experimental animal. The animals had only a basic feed ration compared with the ration used by breeders for pubertal gilts to stimulate sexual processes. This dose restriction therefore did not stimulate early sexual maturity. This fact, together with the stress (animal transport, daily manipulation) was probably the reason that no rut was reported during the period of housing and clinical experiments.
All procedures were carried out in accordance with the protocols approved by the State Veterinary and Food Administration in Bratislava (decision no. 1319/13-221) as well as by the Animal Use Committee at the Institute of Neurobiology, Slovak Academy of Sciences, and in accordance with the EC Council Directive (2010/63/EU) regarding the use of animals in research. All efforts were made to minimize the size of experimental groups and animal suffering.

Experimental groups. In a pilot study three minipigs were used for standardization of the compression technique by testing the U shape/circular aluminum bars, and two other minipigs were assigned for testing of spinal perfusion chambers. These animals were excluded from immunohistochemical, histochemical and behavioral analyses.

Twenty-five animals were randomly divided into six experimental groups: i) control group without surgical intervention $(\mathrm{n}=3)$; ii and iii) spinal cord compression at the $\mathrm{L} 3$ segment induced by $8 \mathrm{~N}(\mathrm{n}=4)$ or $15 \mathrm{~N}(\mathrm{n}=4)$ force; iv and v) groups that underwent spinal cord compression (8N force) and local hypothermia $(5 \mathrm{~h})$ with saline $(\mathrm{n}=4)$ or oxygenated culture medium (DMEM/F12) (n=3); vi) group that underwent spinal cord compression ( $15 \mathrm{~N}$ force) and local hypothermia $(5 \mathrm{~h})$ with saline $(n=7)$. All experimental animals survived for 9 weeks.

Pre-surgical procedures. The minipigs were premedicated with an intramuscular injection of Stresnil (2 mg/kg; Janssen Pharmaceutica, Beerse, Belgium) and Atropin $(0.5 \mathrm{mg} / \mathrm{kg}$; Biotika a.s., Slovenská L'upča, Slovakia), and then anesthetized with Thiopental (10 mg/kg; Czech Pharma, Praha, Czech Republic). Prior to surgery, the precise location of the L3 vertebra was identified using plain X-rays in a lateral projection (Fig. 1) (19). Afterwards the animals were intubated with an endotracheal tube (diameter 5.0-6.0 mm) and the anesthesia was maintained with Sevoflurane (1.5\%; Baxter Czech spol. s r.o., Praha, Czech Republic) mixed with oxygen. For both the surgical procedure and the duration of hypothermia, the analgesia was supported by the administration of Butomidor (0.4 mg/kg; i.v.; Richter Pharma AG, Wels, Austria). Catheters for the administration of infusions and medicaments were inserted bilaterally into the cephalic and/or auricular veins.

Spinal cord compression. The minipigs were placed in a special immobilization apparatus consisting of a basal oval stainless steel platform $(50 \times 90 \mathrm{~cm})$ with four horizontal bars $(2 \mathrm{~cm}$ in diameter) and four vertical bars (size $3 \times 9 \mathrm{~cm}$ and $23 \mathrm{~cm}$ length) (Fig. 1A). The apparatus was placed on a standard operating table. The lumbar portion of the minipigs was fixed in a prone position with the horizontal bars slid bilaterally against the lateral portion of their paravertebral muscles. After disinfection of the lumbar area, a midline skin and subcutaneous fat incision was made from L2 to L4 vertebrae. Paraspinal muscles were retracted to access the L2-L4 laminae and a laminectomy was carried out. The SCI was induced at the L3 segment using a compression device consisting of a computer-controlled stepping motor (ViDiTo, Kosice, Slovakia) and a digital force gauge bridged to a 5-mm diameter circular aluminum bar (Fig. 1B). The computer software (FORCE; ViDiTo) controlling the compression arrangement permitted parameters such 
as compression rate, pressure and force to be obtained (15). To control the uniformity of the impact force, the compression curve was recorded in each animal. After spinal cord compression ( $8 \mathrm{~N}$ or $15 \mathrm{~N}$ force; velocity: $30 \mathrm{~mm} / \mathrm{sec}$.), the paraspinal musculature and skin were sutured with several absorbable stitches and the animals were housed in separated cages to recover.

Preparation of perfusion chamber. Two types of chambers were used. In the initial experiments the closed chamber was fabricated to fit into the injury site. For that, in two animals the site of laminectomy was scanned with an eScan 3D laser scanner (3D Digital Corp., Sandy Hook, CT, USA). Data were then transferred to Rhinoceros 3D software (MCNeel \& Associates, Seattle, WA, USA) where the scanned data were used to finalize a prearranged model chamber, to make the bottom surface of the chamber fit perfectly with the bones over the injury site. The spinal perfusion chamber therefore perfectly fitted the injury site (Fig. 2A). The finalized perfusion chamber was then printed in ABS plastic using a uPrint SE 3D printer (Stratasys, Eden Prairie, MN, USA). The spinal perfusion chamber was fitted with inflow and outflow tubing. However, the closed chamber did not allow cleaning of the spinal spinal dural sac, which was frequently covered with blood clots, preventing contact between the solution and the medullary tissue. For this reason these animals were excluded from the experiment. For all following experiments, an open design of the chamber was prepared. The chamber in the shape of a cone with both ends opened (Fig. 2B and C) was printed from ABS plastic using a uPrint SE 3D printer (Stratasys). The spinal perfusion chamber had ellipse-shaped openings, the lower opening measuring 20x14 mm and the upper one $40 \times 30 \mathrm{~mm}$, and the height of the chamber was $50 \mathrm{~mm}$. The spinal perfusion chamber was fitted with two tubings (inflow and outflow). An individual chamber was fabricated for each animal.

Implantation of perfusion chamber. The perfusion chamber was implanted over the exposed spinal dural sac containing the spinal cord (Fig. 2B and C). The smaller opening was about the size of the laminectomy, so it could be freely placed directly on the dura mater with no additional fixation to surrounding tissue. Only two stitches partially closed the wound, so the skin edges slightly embraced the chamber. No additional pressure was generated, since the weight of the chamber was minimal ( $4 \mathrm{~g}$ ), and the paraspinal muscles and subcutaneous fat layer around conical chamber would lift it, rather than push it down. The contact of the chamber with dura was checked periodically during removal of blood clot from the surface of dura mater through perfusion chamber. No leakage was detected. Since the chamber was placed freely on the dural surface, without any fixture to the spine or paravertebral muscles, the edema which developed after SCI just lifted the chamber, without causing any additional harm.

Hypothermia. Hypothermia with saline or porcine medium (DMEM/F12) consisting of rh-Transferrin $100 \mathrm{mg} / \mathrm{l}$, rh-Insulin $25 \mathrm{mg} / \mathrm{l}$, Glucose $1.56 \mathrm{~g} / \mathrm{l}$, Progesterone $6.3 \mu \mathrm{g} / \mathrm{l}$, Putrescine $16.1 \mathrm{mg} / 1$, Selenium $5.2 \mu \mathrm{g} / 1$ (Cell Guidance Systems Ltd., Cambridge, UK) was carried out locally through each spinal

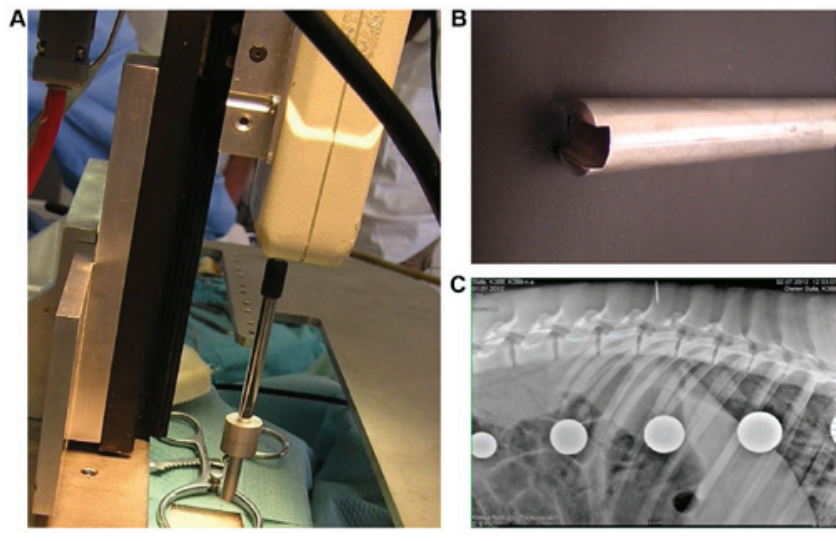

Figure 1. (A) Computer-operated compression apparatus consisting of a computer-controlled stepping motor and (B) digital force gauge bridged to a 5-mm diameter circular bar with U-shaped tip. (C) Correct localization of laminectomy was determined using plain X-rays of the lumbar vertebral column in a lateral projection.
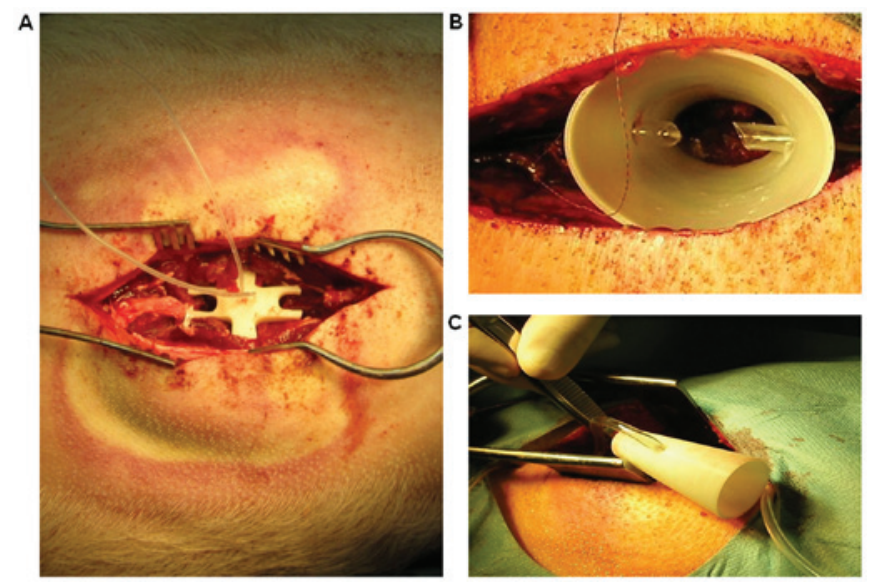

Figure 2. Special spinal perfusion chamber implanted over the exposed spinal dural sac to allow local perfusion of injured tissue with treating solutions. (A) Closed design of the chamber was not useful due to limited contact of solution with spinal tissue, so we used (B and C) an open chamber enabling cleaning and monitoring of the spinal dural sac surface.

perfusion chamber. The inflow tubing of the perfusion chamber was connected to the tank with sterile ice cold $\left(4^{\circ} \mathrm{C}\right)$ perfusion solution. The cooling of the site of injury was initiated $30 \mathrm{~min}$ after SCI. At the beginning the solution drip rate was manually adapted to ensure that the temperature of fluid in the perfusion chamber (just above the SCI) was maintained at $19^{\circ} \mathrm{C}$. Given that cooling was applied locally, the temperature of the solution in the chamber decreased to $19^{\circ} \mathrm{C}$ very rapidly, within 4-5 $\mathrm{min}$. The temperature of solutions in the perfusion chamber was measured at 15 min intervals using a needle-tip thermometer (Omega Bio-Tek, Inc., Norcross, GA, USA) during the whole treatment procedure $(5 \mathrm{~h})$. Continuous inflow of the perfusion solution into the chamber was regulated by a peristaltic pump (Heidolph, BRD, the flow $2 \mathrm{ml} / \mathrm{min}$ ). Outflow tubing was used to drain excess perfusion solution.

To minimize variability in body temperature during hypothermic treatment the minipigs were covered with a thermal blanket. The rectal temperature of each animal was measured at 15 min intervals using a digital predictive thermometer 
Table I. 20-point neurological scale for minipigs .

Scale

Description

0 Complete paraplegia, no movement in joints of hindlimbs, spontaneous defecation and urination

1 Slight movement in one joint (hip joint) of hindlimbs, not capable of sitting and plantar placement of hindlimbs during sitting, spontaneous defecation and urination

2 Slight movement in two joints (hip and knee joint) of hindlimbs, not capable of sitting and plantar placement of during sitting, spontaneous defecation and urination

3 Slight movement in all three joints of hindlimbs, capable of sitting on the side without plantar placement of hindlimbs during sitting, spontaneous defecation and urination

4 Extensive movement in one joint (hip joint) and slight movement in two joints of hindlimbs, capable of sitting on the side without plantar placement of hindlimbs during sitting, spontaneous defecation and urination

5 Extensive movement in twoo joints of hindlimbs and slight movement in third joint of hindlimbs, capable of straight sitting and plantar placement of hindlimbs during sitting, spontaneous defecation and urination

6 Extensive movement in all three joints of hindlimbs, capable of straight sitting and plantar placement of hindlimbs during sitting, spontaneous defecation and urination

7 Extensive movement in all three joints of hindlimbs, not capable of standing up, sweeping with one hindlimb, spontaneous defecation and urination

8 Extensive movement in all three joints of hindlimbs, not capable of standing up, sweeping with both hindlimbs, spontaneous defecation and urination

9 Sweeping with both hindlimbs, not capable of plantar hoof stepping, attempt to hold stand with initial help to stand up

10 Sweeping with both hindlimbs, capable of weigh support with plantar surface of hoof with initial help to stand up only, not capable of walking

11 Capable to stand up itself, not capable to maintain balance, walking with support only (falling back on wall), position only on dorsal surface of hoof

12 Capable to stand up itself on hindlimbs and capable standing, able to walk 1-3 steps with occasional support without forelimb-hindlimb coordination, occasional plantar-hoof stepping

13 Capable of standing up spontaneously and able to take 1-3 steps without support before losing balance, no forelimbhindlimb coordination, frequent plantar-hoof stepping and capable to keep balance between stepping episodes

14 Capable of standing up spontaneously and able to take 3-5 steps, capable to keep balance between stepping episodes with occasional support only, no forelimb-hindlimb coorination, occasional to frequent plantar-hoof stepping

15 Capable of standing up spontaneously and able to take 3-5 steps, capable to keep balance between stepping episodes, frequent plantar-hoof stepping, no forelimb-hindlimb coorination

16 Capable of standing up spontaneously and able to take 5-10 steps, capable to keep balance, frequent plantar-hoof stepping, no or inconsistent forelimb-hindlimb coordination

17 Capable of standing up spontaneously and able to take 5-10 steps, capable to keep balance, frequent plantar-hoof stepping, occasional forelimb-hindlimb coordination

18 Capable of standing up spontaneously and able to take 5-10 steps, capable to keep balance, frequent plantar-hoof stepping, frequent forelimb-hindlimb coordination

Capable of standing up spontaneously and able to take more than 10 steps, capable to keep balance, consistent plantarhoof stepping, frequent forelimb-hindlimb coordination Capable of standing up and walking spontaneously, consistent plantar-hoof stepping, consistent forelimb-hindlimb coordination

Scale $1-8$, the movement of hindlimbs in each individual joint; scale 9-11, the ability to stand up; scale $12-20$, the ability to walk.

with the use of lubricant. Each single rectal measurement took 10-20 sec. When the temperature dropped below $36^{\circ} \mathrm{C}$ the animals were warmed with a stream of warm air (a hair dryer blew warm air below the blanket covering the animal). This heating method was very efficient and maintained the minipigs at normothermia during the whole procedure. The rectal temperature varied during hypothermia from $35.4^{\circ} \mathrm{C} \pm 0.4$ to $36.6^{\circ} \mathrm{C} \pm 0.4$. After finishing the therapy the perfusion chamber was explanted and the incision was closed with absorbable sutures. The animals were housed in separated cages to recover.
Post-operative animal care. Post-operative care included bladder expression twice a day, antibiotic administration (Penstrepten $(0.5 \mathrm{ml} / 30 \mathrm{~kg} /$ day; Biotika a.s.) for 10 days, cleaning the hind limbs and monitoring of skin irritation and development of decubitus ulcers. Abrasions of the tail and perianogenital region occurred in $18 \%$ of animals (3 out of 4) subjected to $15 \mathrm{~N} \mathrm{SCI}$ and 2 out of 7 treated with cold saline after $15 \mathrm{~N}$ SCI. The affected areas (bruises around the tail) were treated twice a day with $0.1 \%$ solution of Rivanol and with Alamycín spray (Norbrook, Newry, Northern Ireland, 
UK) for about one week. The animals survived for an additional 2 months without further complications. Pressure sores or other skin lesions were not observed in the $8 \mathrm{~N} \mathrm{SCI}$ group, $8 \mathrm{~N} \mathrm{SCI}$ animals treated with cold saline or medium, or in the rest of animals after $15 \mathrm{~N} \mathrm{SCI}$ or $15 \mathrm{~N} \mathrm{SCI}$ and treatment.

The 20-point porcine neurological scale to determine hind-limb motor recovery after spinal cord compression. Recently, the porcine neurological motor score was re-graded on a 10-point scale according to Lee et al (20) or a fourteen-point grading scale according to Navarro et al (15). Although both protocols are well validated and easily replicated, the assessment of functional recovery in this study took into principal consideration the hind-limb movement for each individual joint, the capability of each animal to get up by itself, its trunk stability and stepping coordination. The scoring points range from complete paraplegia (zero) to normal ambulation (20 points) and represent three sequential recovery stages which the minipigs attained after SCI and hypothermic treatment. The 20-point neurological scale for minipigs is described in detail in Table I. Porcine neurological motor scores (1-8) represent slight or extensive movement for each individual joint of the hind limbs, points (9-11) represent sweeping and the ability to get up by itself (with or without assistance; these animals were not able to maintain balance), and points (12-20) characterize the ability to walk from a few steps without fore-limb/hind-limb coordination to consistent plantar-hoof stepping and consistent fore-limb/hind-limb coordination (see representative pictures in Fig. 3).

Behavioral assessment. Three weeks before the surgery the minipigs underwent half an hour of training for 5 days/week to walk upon command up and down a rubber mat (width $0.8 \mathrm{~m}$, length $5.2 \mathrm{~m}$ ). One week before SCI the minipigs were videotaped. Post-operative neurological impairment was tested under blinded outcome assessment two days after surgical procedures, and continued weekly for nine weeks of survival. Each minipig was allowed to walk freely on a rubber mat for $10 \mathrm{~min}$ without assistance. A video recording was made of each evaluation. In order to reduce bias, three independent observers were involved in the development of the neurological scale. To blind the outcome assessment, the observers graded the neurological functions independently without knowledge of the treatment. To minimize the variability, each camera record was re-scored by the same three observers. The inter-rater differences in the scores varied max by 1 point. The discrepancies obtained by the raters from two independent assessments were discussed and clarified. Based on direct behavioral observations of neurological recovery and re-scoring the videos we developed our own porcine neurological scale to determine hind-limb motor recovery after spinal cord compression (Table I).

Spinal cord tissue preparation. At the end of survival, the minipigs were deeply anesthetized with an intravenous overdose of thiopental and transcardially perfused with heparinized saline (51), followed by $4 \%$ paraformaldehyde in $0.1 \mathrm{M}$ phosphate-buffered saline (PBS, pH 7.4, 51). The lumbar spinal cord (approximately $10 \mathrm{~cm}$ ) was carefully dissected out,
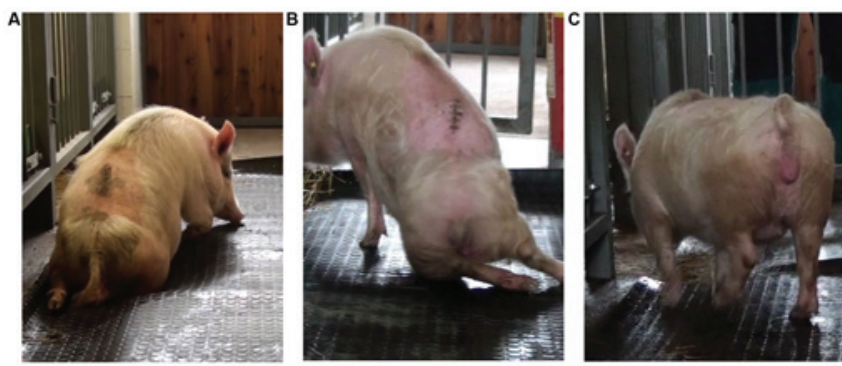

Figure 3. Representative pictures of the porcine behavioral scoring system (points 0-20). (A) Points 1-8 represent the movement of hind limbs in each individual joint. (B) Points 9-11 represent hind-limb sweeping and the animal's ability to stand up by itself. (C) Points 12-20 represent the ability to walk. The neurological scale for minipigs is described in detail in Table I.

cleaned of the dura mater and post-fixed in the same fixative overnight. Next day, the spinal cord was cut into one $1 \mathrm{~cm}$ block (lesion site) and three $1 \mathrm{~cm}$ blocks caudally $(-1,-2,-3)$ as well as cranially $(+1,+2,+3)$ from the epicenter of injury. In the next step, the spinal cord pieces were cryopreserved in a solution of $30 \%$ sucrose in PBS at $4^{\circ} \mathrm{C}$ for $48 \mathrm{~h}$. Afterwards, each tissue block was cut into transverse serial sections (30 $\mu \mathrm{m}$ thick) on a cryostat (Leica Microsystems, Wetzlar, Germany) and stored in PBS. Transverse serial sections were mounted on slides and were processed in accordance with immunofluorescence and histochemical procedures.

Immunofluorescence staining. For processing of neurofilament immunoreactivity, the slices taken from the rostral and caudal blocks were incubated in blocking solution consisting of 5\% normal goat serum in $0.1 \mathrm{M}$ phosphate-buffered saline (PBS) with $0.3 \%$ Triton-X 100 for $1 \mathrm{~h}$ at room temperature. After blocking the slices were incubated overnight at $4^{\circ} \mathrm{C}$ with primary monoclonal mouse antibody anti-neurofilaments (Pan-Axonal Neurofilament Marker SMI312, 1:1,000; cat. no. ab24574; Abcam, Cambridge, UK) diluted in $0.1 \mathrm{M}$ PBS with 5\% normal goat serum and 0.3\% Triton-X 100 . Next day, the sections were washed three times in $0.1 \mathrm{M}$ PBS for $10 \mathrm{~min}$ and incubated with secondary anti-mouse antibody (Rhodamine Red $^{\mathrm{TM}}-\mathrm{X}$ conjugated AffiniPure goat anti-mouse IgG, 1:200; cat. no. 115-295-003; Jackson ImmunoResearch Laboratories, Inc., West Grove, PA, USA) in Tris-buffered saline containing $0.1 \mathrm{M}$ phosphate buffer $(7.4 \mathrm{pH})$ with $5 \%$ normal goat serum and $0.3 \%$ Triton-X 100 for $90 \mathrm{~min}$ at room temperature. The sections were finally rinsed in 0.1 M PBS and cover-slipped with Fluoromount-G (cat. no. 21644; Serva Electrophoresis. GmbH, Heidelberg, Germany). Microphotographs were taken using a fluorescence microscope (Olympus BX51/BX52; Olympus, Hamburg, Germany) with an Olympus DP50 digital camera coupled with a computer equipped with Olympus DP Image software (version 3.1). Neurofilament immunoreactivity was evaluated using the Image J graphics software. SMI312 labeled axons were counted by a blinded observer using a $20 \mathrm{x}$ lens in a $800 \times 600 \mu \mathrm{m}$ area in three specific regions of the white matter (dorsal, lateral and ventral columns) (Fig. 4). The number of neurofilaments (expressed as percentage of $\mathrm{NF} / \mathrm{mm}^{2}$ ) was evaluated bilaterally from five randomly-selected transverse slices of each spinal cord block. 


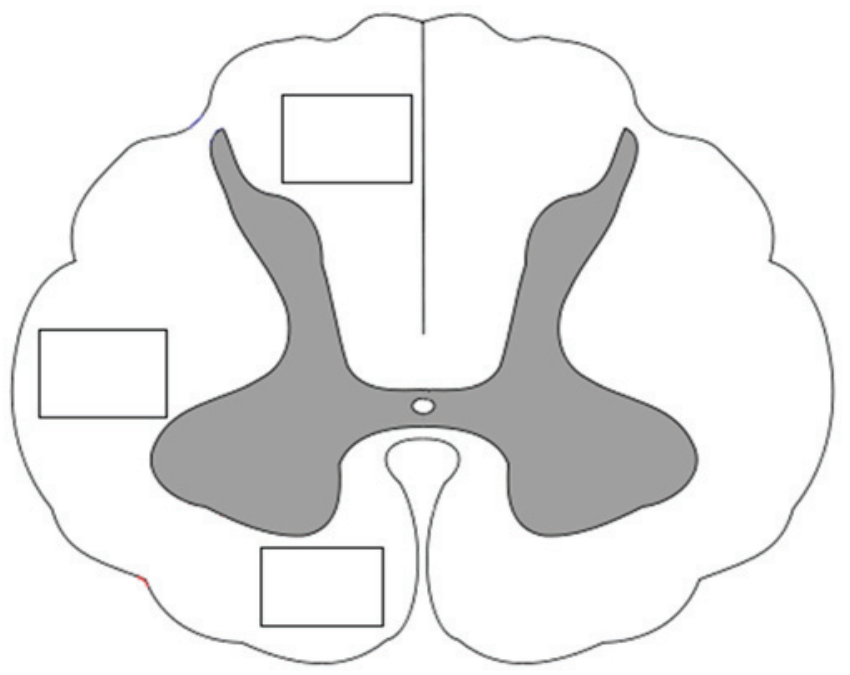

Figure 4. Representative picture of the spinal cord showing localization of three areas of interest taken for quantitative analysis of neurofilaments.

Histological analyses. The spinal cord slices (lesion site and $1 \mathrm{~cm}$ blocks in rostral and caudal directions) were stained according to the protocol for luxol fast blue and cresyl violet staining to recognize myelinated fibers in white matter and the residual preserved tissue. The percentage of total volume of preserved spinal tissue was evaluated using an Olympus (BX51/BX52) light microscope and ImageJ software.

Data acquisition and statistical analysis. The results from neurological analyses were assessed in a blinded manner and statistically evaluated using Kruskall-Wallis or Mann-Whitney tests. All immunohistochemical and histological data were analysed in a blinded manner using one-way ANOVA test. $\mathrm{P}<0.05$ was considered to indicate a statistically significant difference. All outcomes were expressed as mean values with standard errors of the mean (SEM). The correlation between degree of neurological outcome, number of neurofilaments and gray and white matter preservation was assessed using the Pearson correlation test. The test was used to evaluate the coefficient of determination $\mathrm{r}^{2}$.

\section{Results}

Behavioral outcomes. Recovery of motor function was assessed using a 20-point scoring system representing the recovery stages which the minipigs attained after SCI and local hypothermia with saline or porcine medium (DMEM/F12). In the first two weeks after SCI (impact force $8 \mathrm{~N}$ and $15 \mathrm{~N}$ ) the behavioral status of each animal was very low in both experimental groups (Fig. 5). These animals showed total loss of locomotor function in the hind-limbs or just slight movement in hip and knee joints (score $0-2$ ). The outcome improved notably from the 2 nd to 5 th week in the group of minipigs which underwent impact with $8 \mathrm{~N}$ force. Hind-limb movement in this group of animals recovered spontaneously from the 5th week and later on, during the last two weeks the outcome was stabilized. In the 9th week the motor score reached 10.8 \pm 2.2 . In the animals which underwent $15 \mathrm{~N}$ impact, very slow spontaneous functional recovery was observed. At the end of the survival time the mean score for motor outcome in this group was graded $6.3 \pm 0.5$.

Animals treated with hypothermic saline perfusion following $8 \mathrm{~N}$ compression demonstrated continuous slight improvement during the whole survival period (Fig. 6A). Although the difference in outcome between the two groups (SCI vs. SCI and hypothermia with saline) was not significant at the end of the survival period, the improvement after treatment exceeded compression by 3 scoring points $(10.8 \pm 2.2 \mathrm{SCI}$; $13.7 \pm 1.8 \mathrm{SCI}$ and hypothermia with saline). The saline-treated animals were able to take 1-3 steps with initial help to stand up and keep balance between stepping episodes, but the movement was without fore-limb/hind-limb coordination. Surprisingly, this group of animals had substantially better recovery rate than the minipigs treated with hypothermia using porcine medium (DMEM/F12) containing a supplement of nutrients. During the first post-injury week, no animal treated with $4^{\circ} \mathrm{C}$ medium recovered and complete absence of movement in the hind-limbs or at most slight movement in only one joint was observed. Up to the end of the survival period the medium-treated minipigs showed lower motor function than untreated animals ( $8 \mathrm{~N}$ compression) and at the 9th week the motor score reached only $9.0 \pm 2.3$ points. The behavioral study also showed that recovery in the $15 \mathrm{~N} \mathrm{SCI}$ group treated with cold saline for $5 \mathrm{~h}$ was only slightly better compared with the untreated group (Fig. 6B). From the 4th to 9th week the minipigs treated with $4^{\circ} \mathrm{C}$ saline improved their outcome over compression only by 1 scoring point. This group of animals was not capable of standing up, but we noted sweeping with one hind limb.

Quantitative analysis of neurofilament immunoreactivity. The immunoreactivity for neurofilaments was strong in the dorsal funiculi compared to other white matter columns, and was equally distributed throughout the lateral and ventral column axons of control animals (Fig. 7). Nine weeks after SCI axons in the white matter funiculi were weakly stained with various degrees of staining. Quantitative analysis showed significant decrease in NF intensity generally in all rostral and caudal segments, with the lowest number of NF-IR profiles near the epicenter of injury ( +1 and -1 segments) (Figs. 8 and 9). In the group which underwent 15N SCI we found the most striking alterations at the -1 segment in dorsal and lateral columns (38, 58\%) (Fig. 9). Hypothermia with saline significantly increased the number of neurofilaments in each white matter column, with the most extensive improvement seen in the lateral funiculi (Figs. 8 and 9). The return of NF staining appeared greater in segments $(-1,-2,-3)$ below the SCI than in the rostral segments. Extensive improvement was also noticed in the dorsal column, displaying a similarly high proportion of NF staining in the caudal segments in saline-treated minipigs. In contrast, treatment of injured spinal cord with hypothermic porcine medium (DMEM/F12) solution demonstrated no significant increase in neurofilament intensity in any of white matter columns in comparison with the compression group (Fig. 8).

Histological assessment. Luxol fast blue combined with cresyl violet staining of white and gray matter produced a marked contrast between intact and damaged spinal cord tissue. 


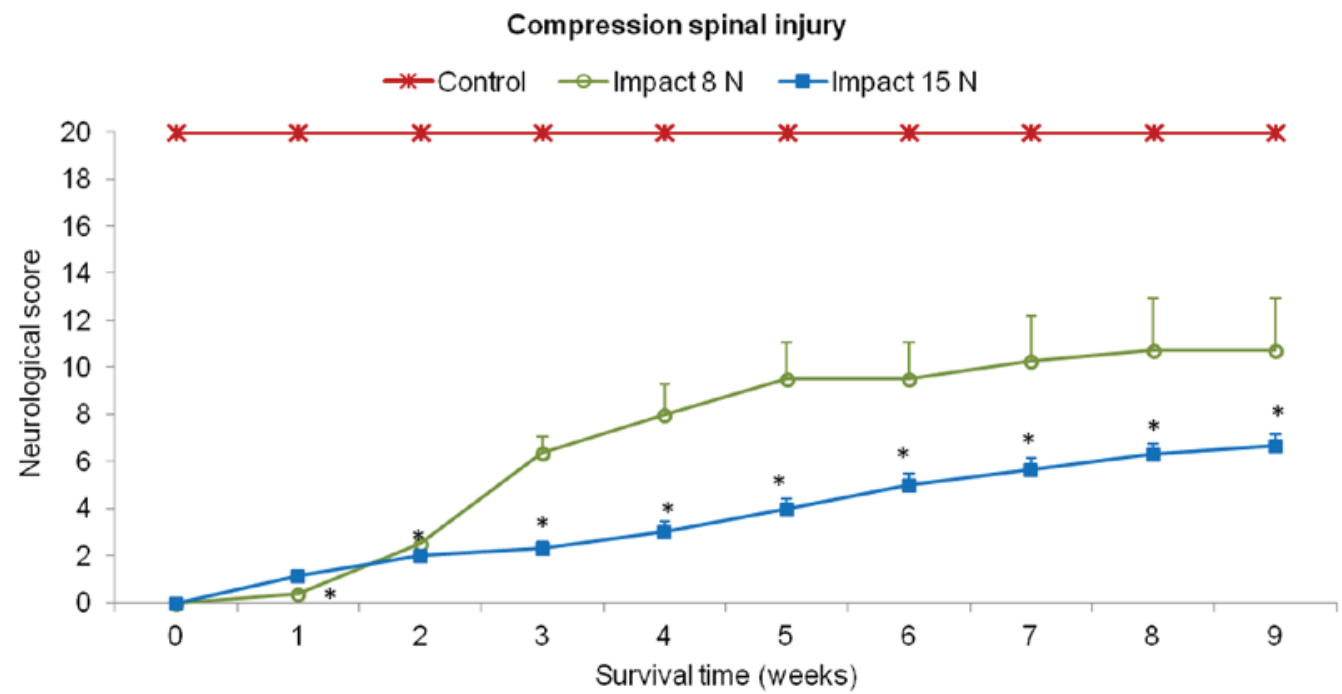

Figure 5. Graph representing motor function recovery analyzed in control animals and those after L3 spinal cord compression ( $8 \mathrm{~N}$ force impact; $15 \mathrm{~N}$ force impact) using the porcine behavioral scale ( 0 , complete paraplegia with loss of hind-limb movements; 20 , normal ambulation and balance) during nine weeks of survival. Kruskall-Wallis testing revealed statistically significant differences between control and spinal cord compression groups ( $\mathrm{P}<0.05)$. Data are given as means \pm SEM ( $n=3$ in control group, $n=4$ in SCI groups). SCI, spinal cord injury.
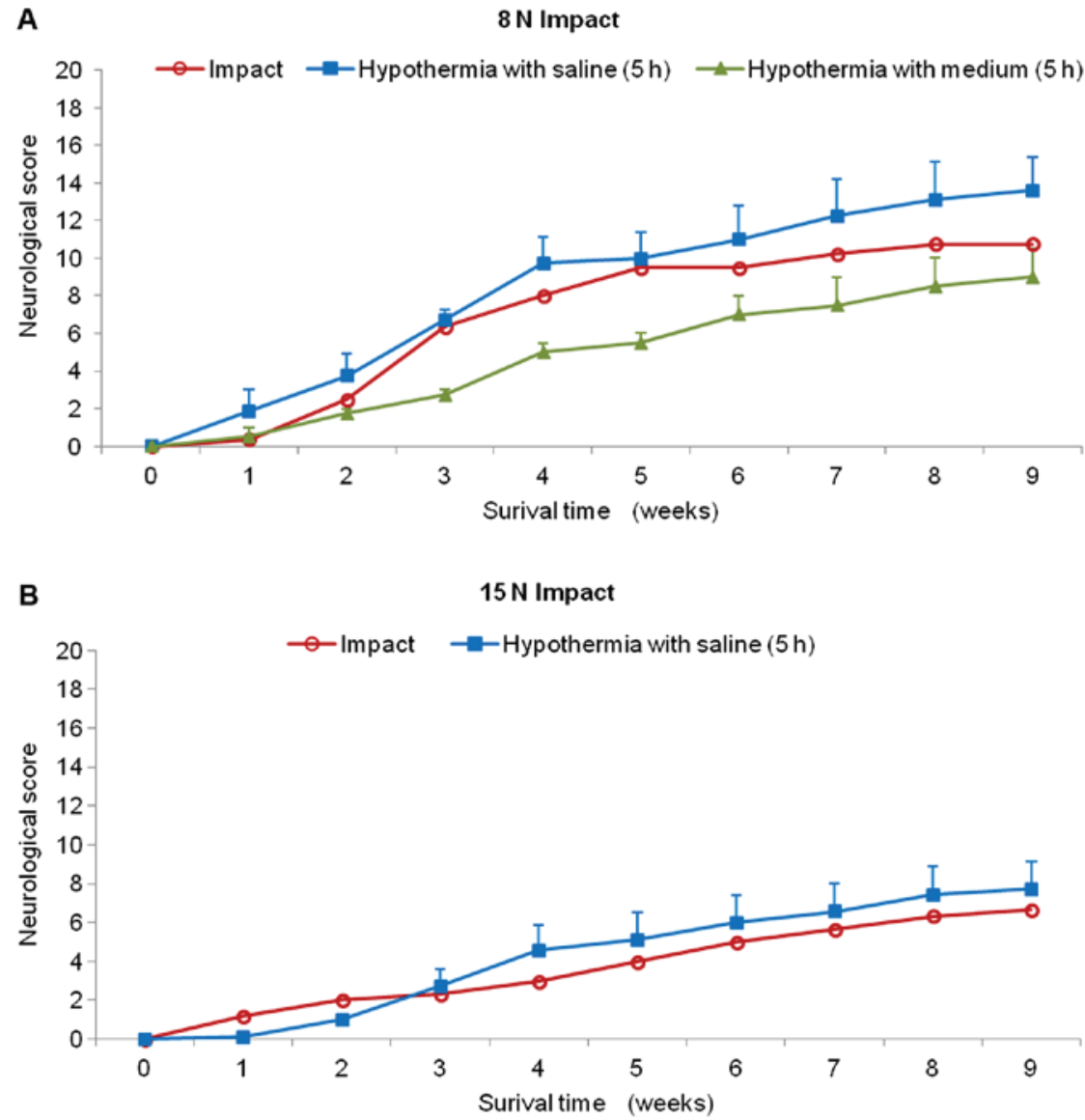

Figure 6. Locomotor function recovery assessed using a 20-point porcine scale in minipigs after undergoing L3 spinal cord compression (force 8N in A; force $15 \mathrm{~N}$ in B) and treatment with local hypothermia (saline or oxygenated culture medium DMEM/F12 in A; saline in B). All experimental animals survived for nine weeks. Data are given as means \pm SEM $(n=3-7)$.

Histopathological assessment of transverse serial sections showed congruous loss of myelin sheaths after trauma inflicted with $8 \mathrm{~N}$ as well as $15 \mathrm{~N}$ force and nine weeks of survival. Tissue degradation was visible in white as well as gray matter in both compression groups. Major spinal tissue destruction occurred at the lesion epicenter and in segments near the site of injury (Fig. 10). The lesion area extended in both directions cranially and caudally from the epicenter. With increased compression 

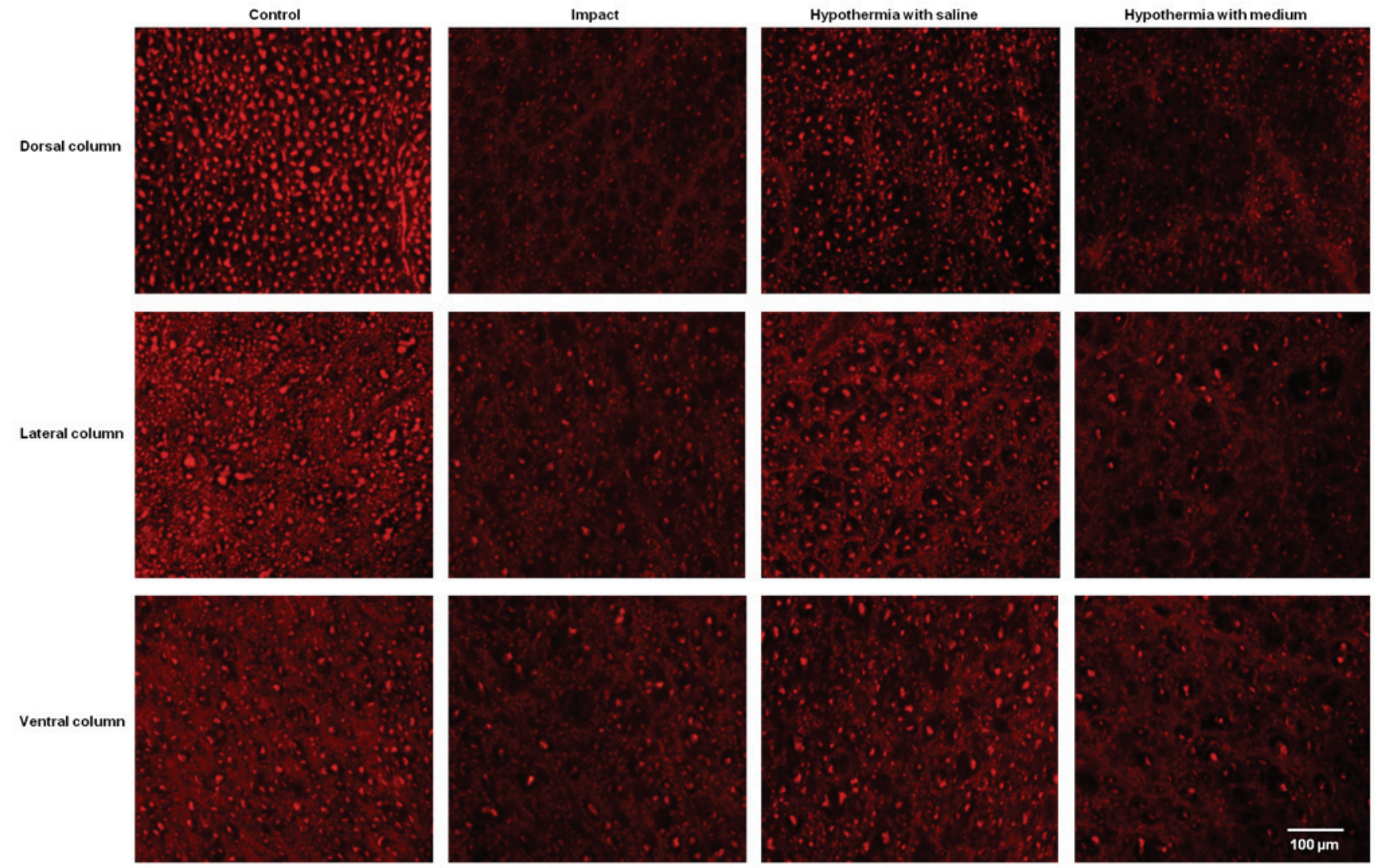

Figure 7. Microphotographs showing neurofilament immunoreactivity in the dorsal, lateral and ventral columns of the lumbar spinal cord (segment -1). Spinal sections were taken from the control group, the group with $8 \mathrm{~N}$ - force impact injury and the group treated with local hypothermia with saline or porcine medium $\mathrm{DMEM} / \mathrm{F} 12$ for $5 \mathrm{~h}$ after spinal cord injury. Scale bar $=100 \mu \mathrm{m}$.

of the spinal cord the structural loss of spinal tissue became greater.

In both compression groups, the gray matter preservation at the lesion site was quite low (53 and 33\%). The preservation was significantly higher in saline-treated animals in $+3,+1$, -1 and -2 segments, compared with untreated $8 \mathrm{~N}$ SCI minipigs, and almost no gray matter loss was seen in +3 and -2 segments. In the animals which underwent $15 \mathrm{~N}$ force impact and were treated with saline hypothermia, the improvement in gray matter preservation was significant in the caudal $(-1,-2)$ segments (Fig. 11).

In the white matter columns, the volume of preserved tissue after SCI $(8 \mathrm{~N}$ and $15 \mathrm{~N})$ at the lesion site was less $(28-41 \%)$ (Figs. 12 and 13) than in the gray matter. Low percentages of preserved white matter was also detected in the section (-1) of the lateral (39\%) and dorsal column (45\%) after 15N SCI, while after $8 \mathrm{~N}$ SCI the preservation in the same segments was better and in the 9th week of survival it reached 50 and $57 \%$ respectively. Hypothermia with porcine medium solution produced only minimal difference between untreated and treated animals (Fig. 12). Quite different results were noted after perfusion of the injured spinal cord with $4^{\circ} \mathrm{C}$ saline. We noted white matter preservation in the rostral and caudal sections of each white matter column, with substantial protection seen around the lesion site in the dorsal column. The most significant attenuation of white matter damage as a whole was seen after SCI $(8 \mathrm{~N})$ treatment in the lateral column. In the $15 \mathrm{~N}$ compression group the most effective percentage of preserved white matter was noted in the dorsal column at the site of injury and caudally $(-1,-2)$ from the lesion site. The differences in preserved white matter between groups are shown in Figs. 12 and 13.

Correlative analyses between behavioral outcome, neurofilament immunoreactivity and histological assessment. To determine the relationship between the histological damage to the spinal cord and behavioral outcome in animals treated with saline for $5 \mathrm{~h}$, we utilized both SCI groups ( $8 \mathrm{~N}, 15 \mathrm{~N}$ force). As shown in Fig. 14, the number of neurofilaments counted in the white matter positively correlated with behavioral recovery. Similarly, positive correlation was observed between behavioral score and cumulative white and grey matter preservation.

Correlative analysis between the extent of axonal loss quantified in the dorsal, lateral or ventral funiculi and the degree of neurological dysfunction demonstrated positive correlation in the lateral funiculi with values $r^{2}=0.4691$ in the rostral section, $\mathrm{r}^{2}=0.4992$ at the epicenter of injury, and a relatively high degree of correlation $\mathrm{r}^{2}=0.8164(\mathrm{P}<0.05)$ in the caudal section. Significant correlation was also found in the dorsal column, but only in the rostral section $\left(\mathrm{r}^{2}=0.9511 ; \mathrm{P}<0.05\right)$ (Fig. 15). Measurements of gray matter preservation (lesion site, and caudally as well as cranially from the epicenter of injury) significantly correlated with the neurological score (Fig. 16).

\section{Discussion}

Is the preservation of the lesion site in the minipig SCI model a predictor of functional outcome? Hypothermic treatments have been reported to be beneficial for some 

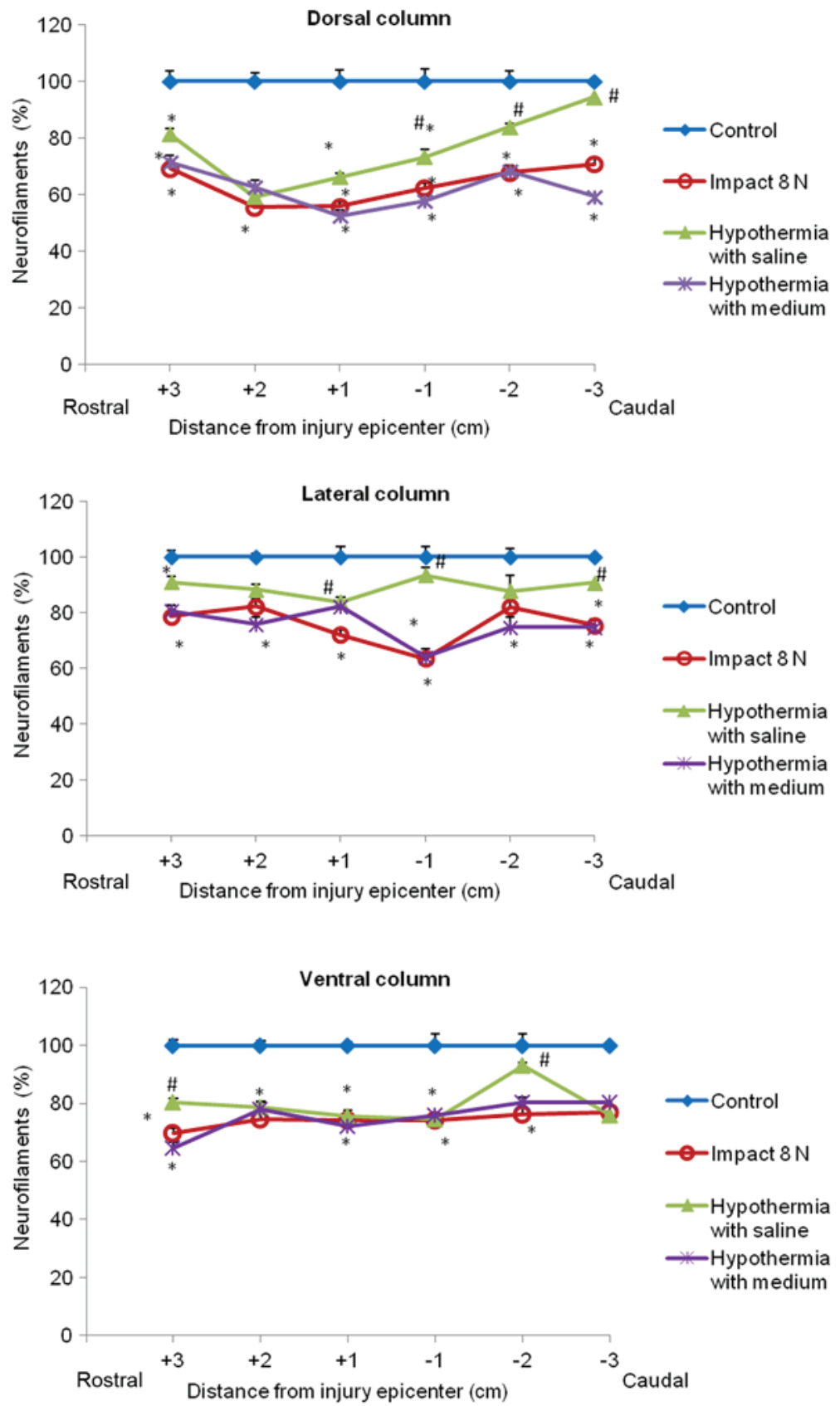

Figure 8. Graph representing the number of neurofilaments (expressed as percentage of $\mathrm{NF} / \mathrm{mm}^{2}$ ) in three areas of interest (dorsal, lateral and ventral columns) in control animals, those after spinal cord compression ( $8 \mathrm{~N}$ force impact) and treatment with cold saline or cold porcine medium DMEM/F12. All experimental animals survived for nine weeks. Asterisks indicate significant differences between control and spinal cord compression groups without treatment $($ ( $\mathrm{P}<0.05)$. The sharp symbols indicate significant differences between treated and untreated animals $\left({ }^{\#} P<0.05\right)$. Data are given as means \pm SEM $(n=3-4)$.

recovery of sensory or motor function in a variety of animal SCI models (21-25). Although animal studies of SCI have revealed consistent neuroprotective effects of hypothermia applied either locally or systemically (26), further experimental/clinical studies are required to determine the optimal cooling parameters (therapeutic window, optimal temperature and duration) and to test the effectiveness of hypothermic therapy in individuals afflicted with this devastating neurological deficit. In the present study, the computer-controlled compression device produced graded contusion lesions at L3 level with two different degrees of tissue preservation and functional recovery, depending upon pre-set impact parameters. Nine weeks after SCI there was a large contusion lesion at the epicenter, with $47 \%$ reduction of the gray matter after $8 \mathrm{~N}$ force impact and $67 \%$ reduction in the $15 \mathrm{~N}$-force SCI group. Early and intense local hypothermia applied 30 min after SCI in the epidural space increased the gray matter preservation at the lesion site in both SCI groups by $\sim 11 \%$. It seems that neurons whose axons have been damaged within a few cell diameters of the cell body upregulate the regeneration-associated genes (27) and may lead to partially enhanced plasticity and reorganization of preserved neuronal circuits. Yoshitake et al (28) demonstrated the neuroprotective effects of local epidural cooling in pigs which underwent ischemic injury. The hypothermic group showed better recovery of somatosensory evoked potentials, 

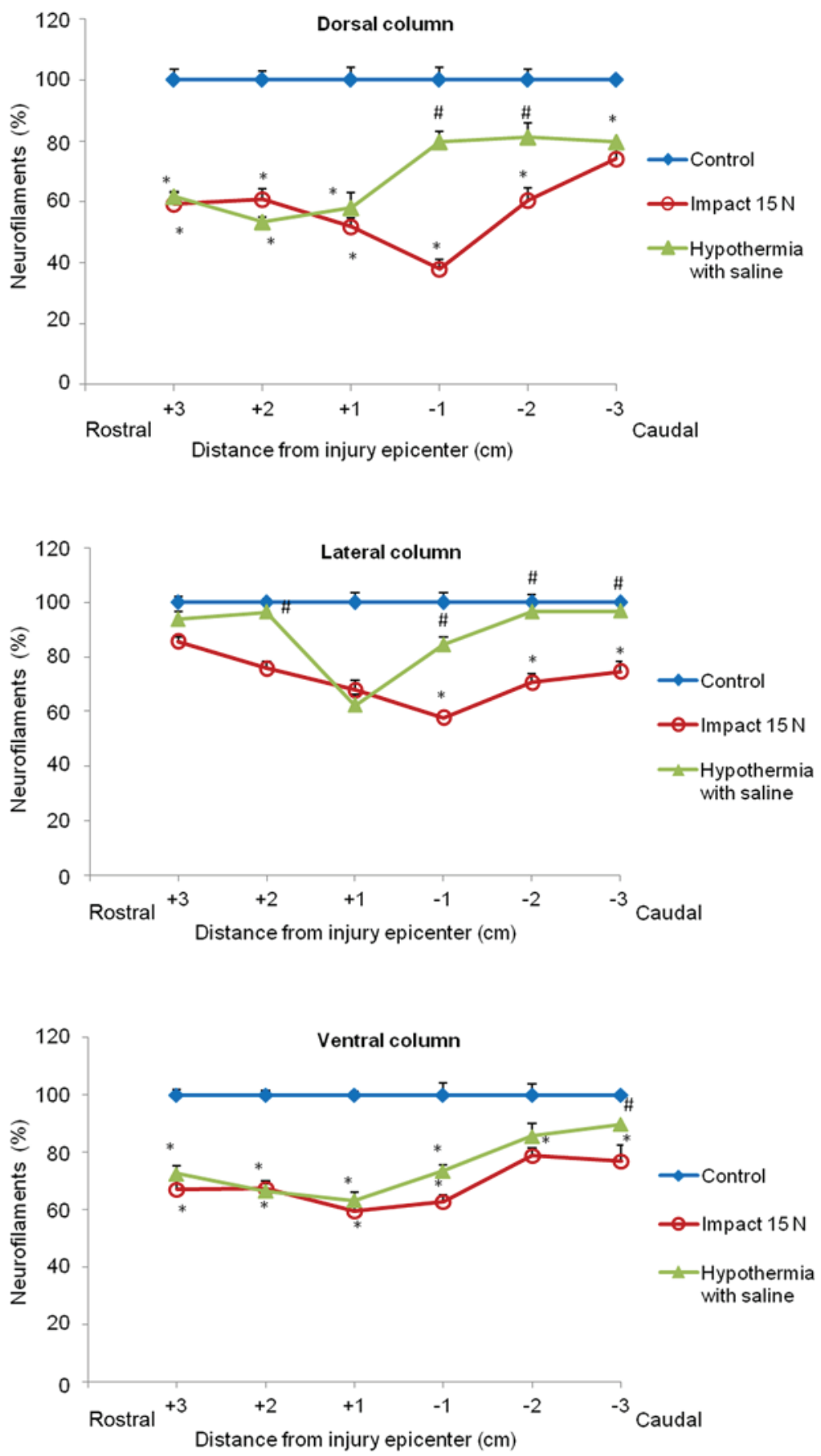

Figure 9. Number of neurofilaments (expressed as percentage of $\mathrm{NF} / \mathrm{mm}^{2}$ ) in the dorsal, lateral and ventral columns in control animals, those after spinal cord compression ( $15 \mathrm{~N}$ force impact) and treatment with cold saline. All experimental animals survived for nine weeks. Asterisks indicate significant differences between control and spinal cord compression groups without treatment $\left({ }^{*} \mathrm{P}<0.05\right)$. The sharp symbols indicate significant differences between treated and untreated animals $(" \mathrm{P}<0.05)$. Data are given as means \pm SEM $(\mathrm{n}=3-7)$.

behavioral ability and increasing numbers of motor neurons in the anterior horn.

Earlier studies documented strong correlation between neurological outcome and the extent of axonal dysfunction at the lesion epicenter (15,26,29-31). Dimar et al (32) reported that local hypothermic treatment at $19^{\circ} \mathrm{C}$ for $2 \mathrm{~h}$ improved neurological function in the rat spinal cord compression model, and indicated that directly-applied hypothermia may be beneficial in preventing injury secondary to ischemic cellular damage. However, the authors failed to find any improvement in the more severe contusion model. In this study, local perfusion of the lesion epicenter with cold saline $\left(19^{\circ} \mathrm{C}\right.$ just above the lesion) produced evident protection of white matter integrity in the dorsal columns (improvement by 13-15\%) with minimal $4-7 \%$ preservation of tissue in the lateral and ventral columns. We speculate that, as in other studies, a small increase in axonal survival at the lesion epicenter may be one of the parameters which enable improvement in neurological function. Nout et al (33) reported that the lesion size per se is not a strict predictor of functional outcome. Our results fully support this suggestion, showing that early local saline hypothermia after $8 \mathrm{~N}$ SCI partially improved tissue integrity 

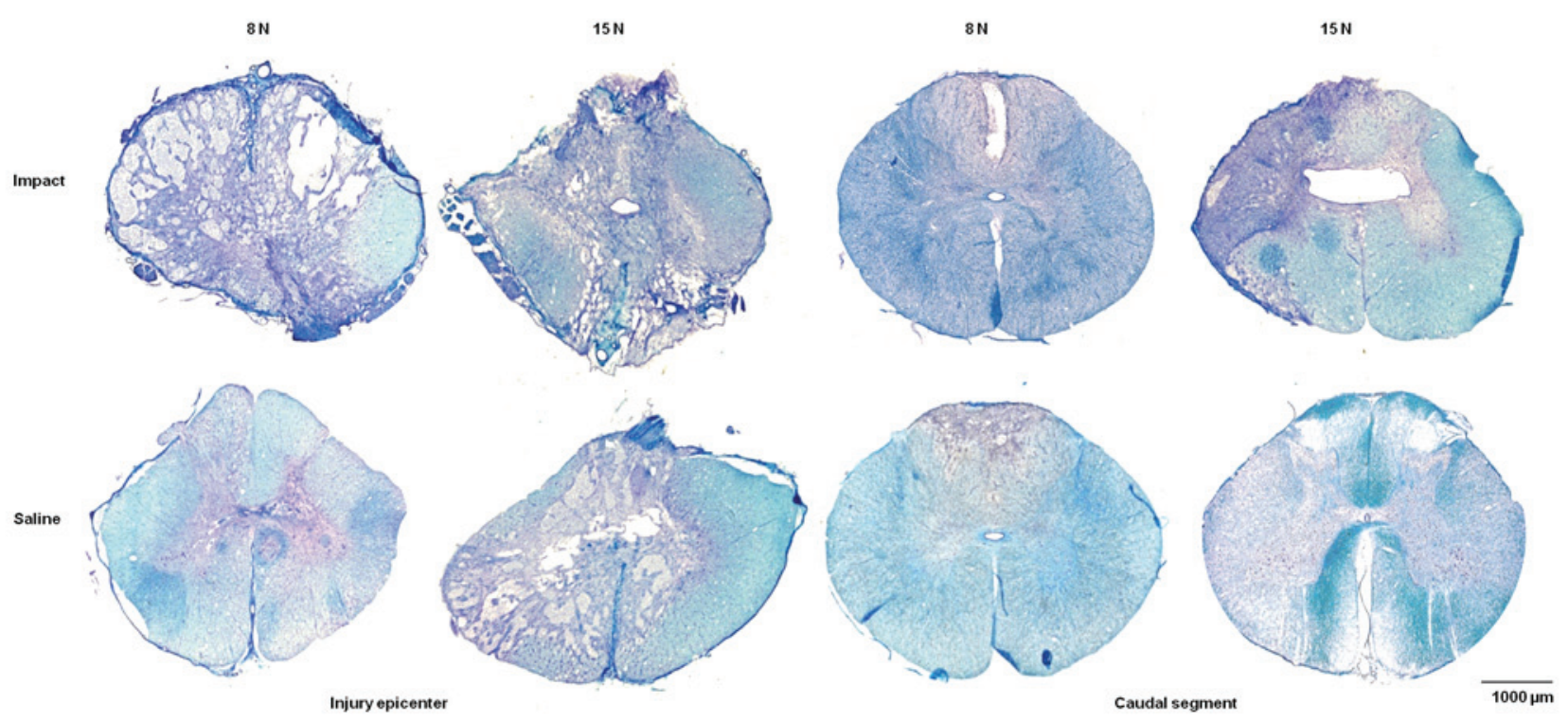

Figure 10. Transverse spinal cord sections taken from the epicenter of injury and caudal segments (-1) after L3 compression ( $8 \mathrm{~N}$ and $15 \mathrm{~N}$ force impact) and treatment with cold saline $(5 \mathrm{~h})$. Animals after SCI and hypothermia survived for nine weeks. Histological sections processed with Luxol fast blue and cresyl violet showed substantial reduction of cavity size at the lesion site and caudally to the epicenter of injury after SCI ( $8 \mathrm{~N}$ impact force)+local hypothermia. SCI ( $15 \mathrm{~N}$ impact force) and local hypothermia with saline show only partial white and gray matter preservation. Scale bar, $1,000 \mu \mathrm{m}$.
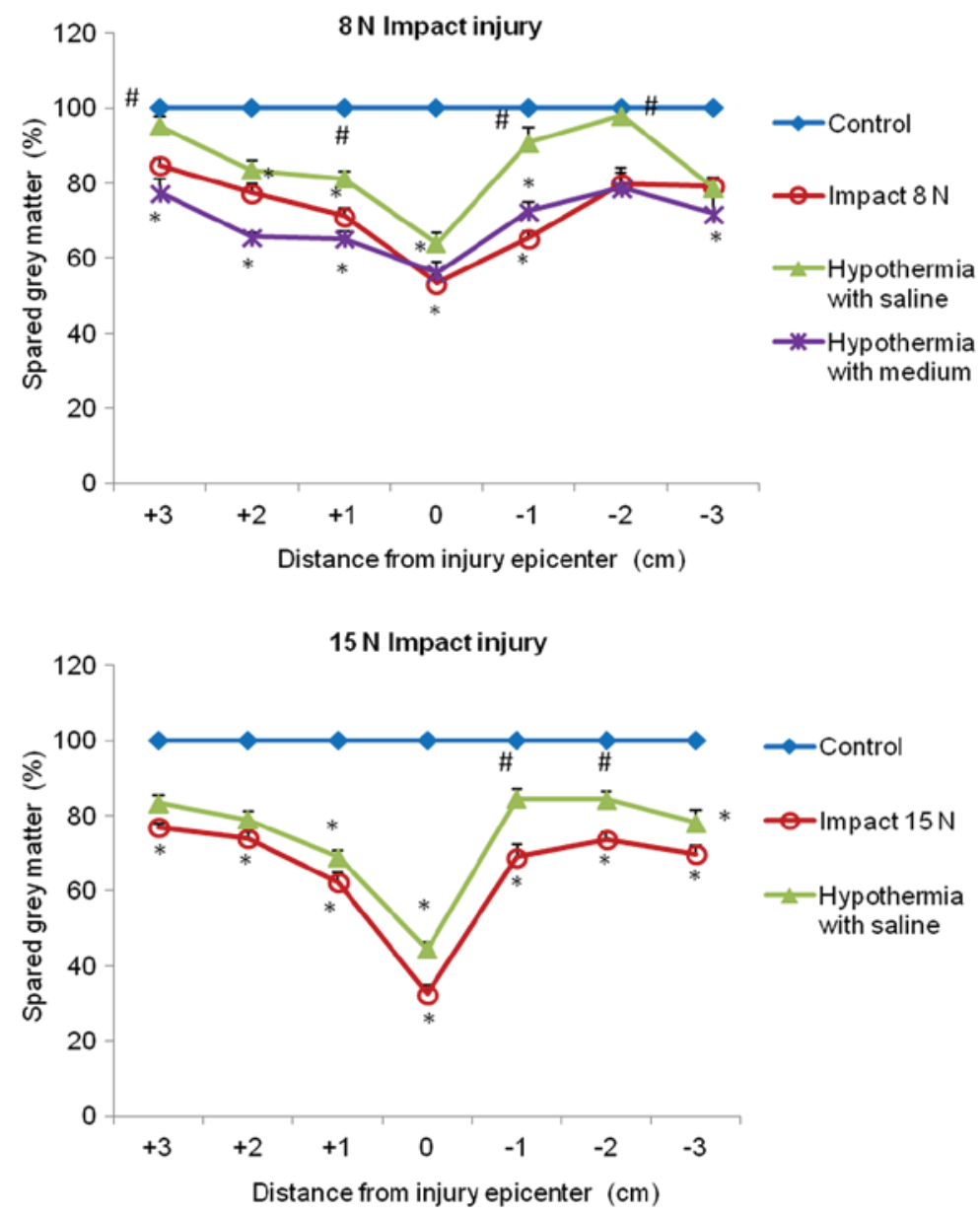

Figure 11. Quantitative analysis of gray matter preservation performed on transverse sections dyed with Luxol fast blue and cresyl violet staining. The percentage of preserved gray matter was calculated in the control group, in animals with $8 \mathrm{~N}$ and $15 \mathrm{~N}$ force spinal cord injury and after treatment with cold saline or oxygenated culture medium DMEM/F12. Experimental animals survived for nine weeks. Asterisks indicate significant differences between control and spinal cord compression groups without treatment $(" \mathrm{P}<0.05)$. The sharp symbols indicate significant differences between treated and untreated animals $\left({ }^{\#} \mathrm{P}<0.05\right)$. Data are given as mean \pm SEM $(\mathrm{n}=3-7)$. 

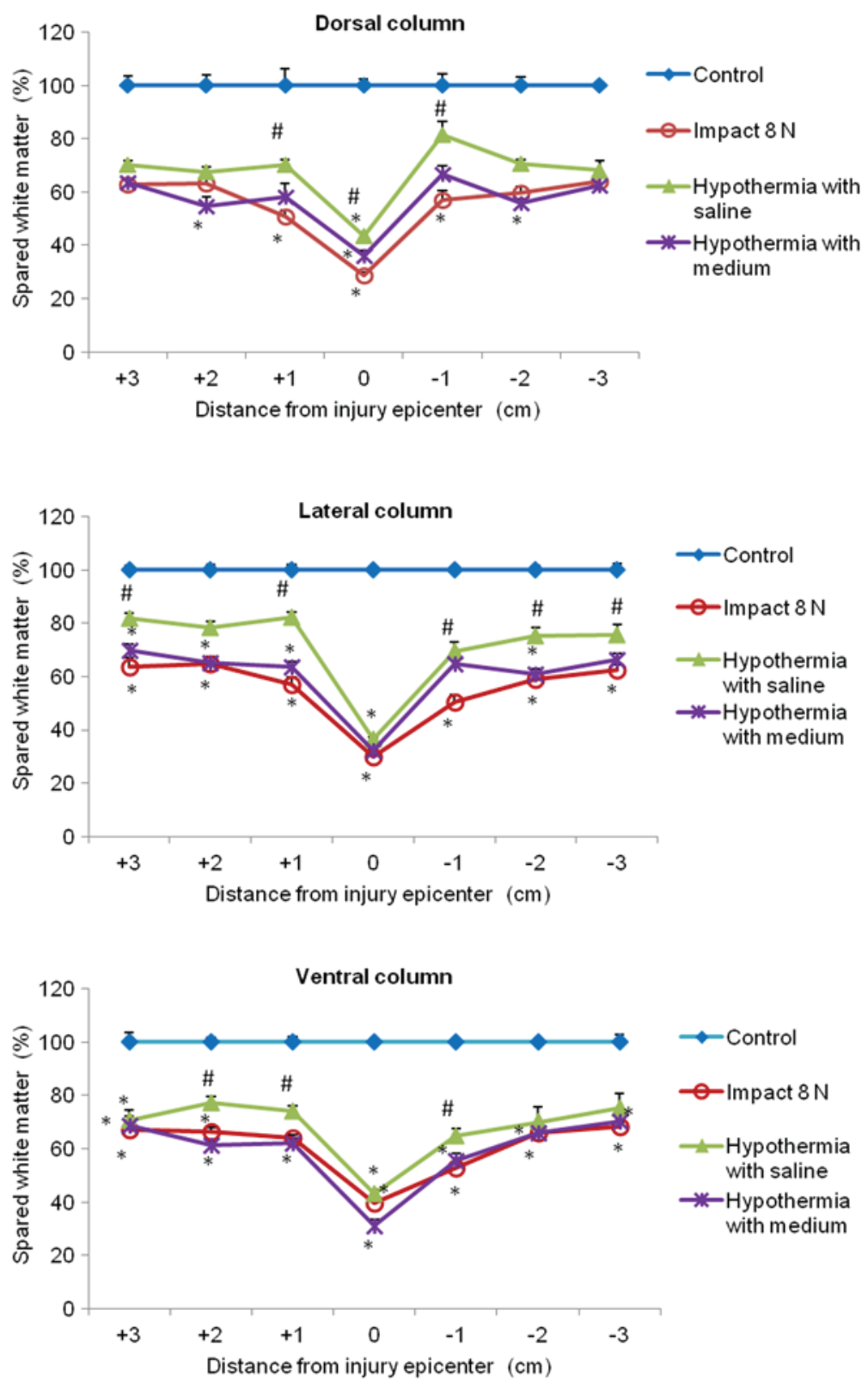

Figure 12. Analysis of preserved white matter in three areas of interest (dorsal, lateral and ventral columns). The percentage of preserved white matter was calculated in control animals, in the group after spinal cord injury ( $8 \mathrm{~N}$ force impact) and in the group after injury and treatment with cold saline or oxygenated culture medium DMEM/F12. Experimental animals survived for nine weeks. Asterisks indicate significant differences between control and spinal cord compression groups without treatment $(" \mathrm{P}<0.05)$. The sharp symbols indicate significant differences between treated and untreated animals $\left({ }^{n} \mathrm{P}<0.05\right)$. Data are given as means \pm SEM $(n=3-7)$.

at the epicenter of SCI (by $11 \%$ in gray matter and 15, 7 and $4 \%$ in the dorsal, lateral and ventral columns respectively), and that this improvement was essential for modulation of the key spinal microcircuits leading to better functional outcome. However, no functional improvement was seen in the more severe $15 \mathrm{~N}$ SCI group treated with saline, although the preservation of both gray and white matter at the epicenter was very similar in both $8 \mathrm{~N}$ - and $15 \mathrm{~N}$-force experimental groups. This finding raises the question to what extent the spinal cord must be preserved.

Fawcett (34) described in his review that in order to produce a two-spinal level improvement in motor function, corticospinal and other descending axonal pathways must regenerate over two or more spinal segments, or for around
2-3 cm. Although extensive spinal relay circuits exist in rodents, the extent to which this circuitry exists and is active in large animal models and primates is unknown and is still controversial. We document that rostro-caudal tissue integrity causing interconnections throughout the lesion site is needed for better neurological outcome in the minipig SCI model.

Integrity of the tissue surrounding the lesion is essential for functional recovery. We attempted to find out to what extent the spinal cord might be saved/repaired after hypothermia, which could be beneficial to minipigs with SCI. For a patient with cervical SCI a return of function over even one segment would improve their quality of life, while return of function over three or four spinal segments would transform it $(34,35)$. To address 

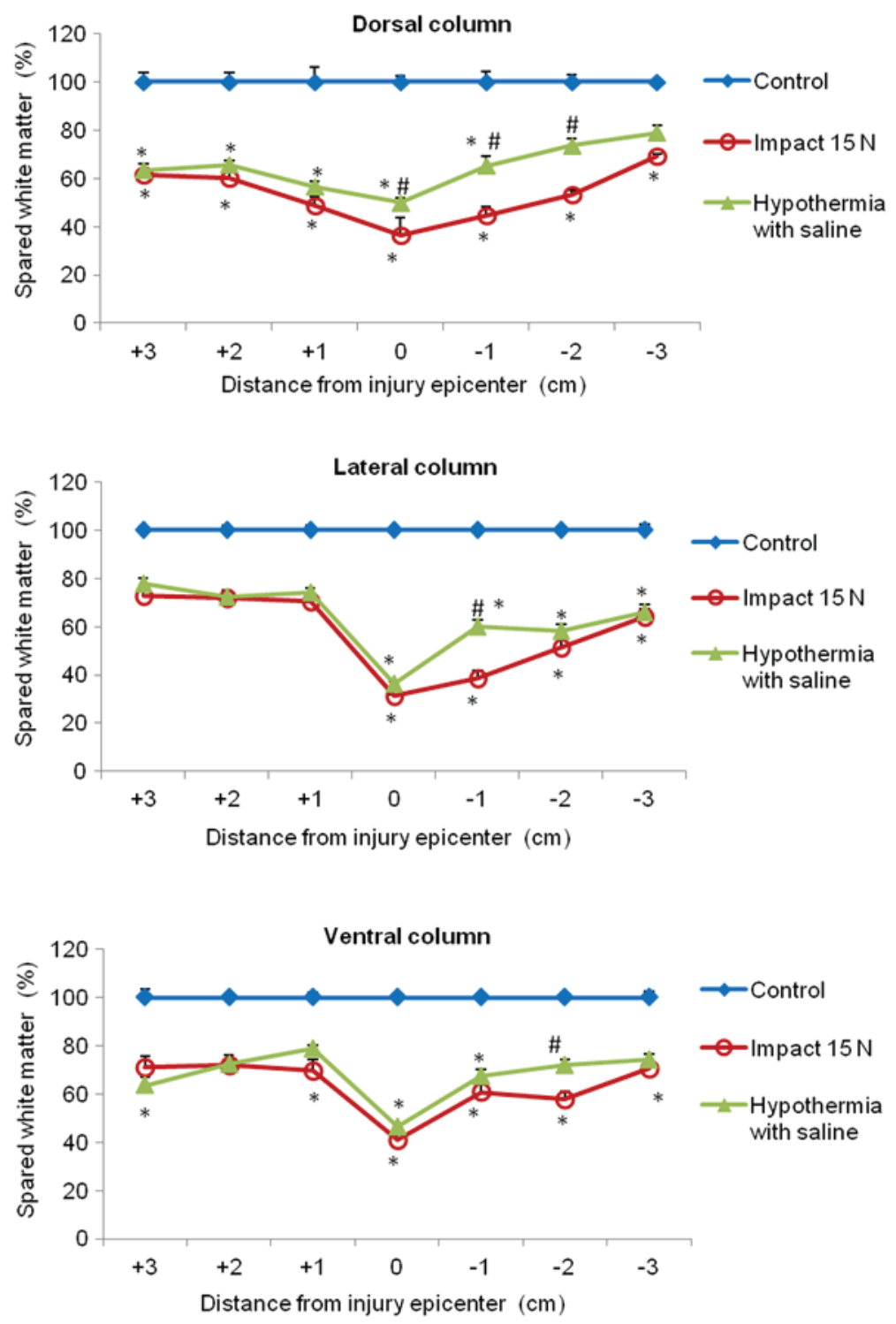

Figure 13. Analysis of preserved white matter in three areas of interest (dorsal, lateral and ventral columns). The percentage of preserved white matter was calculated in control animals, those after spinal cord injury ( $15 \mathrm{~N}$ force impact) and after treatment with cold saline. Experimental animals survived for nine weeks. Asterisks indicate significant differences between control and spinal cord compression groups without treatment ( $\mathrm{P}<0.05)$. The sharp symbols indicate significant differences between treated and untreated animals $\left({ }^{\#} \mathrm{P}<0.05\right)$. Data are given as means $\pm \mathrm{SEM}(\mathrm{n}=3-7)$.

the potential effect of hypothermic treatment we systematically analyzed and quantified the histopathological changes in the spinal sections (rostral $+3,+2,+1$ and caudal $-1,-2,-3$ ) which are likely to be affected by secondary injury. Although the promoting of morphological preservation/recovery was detected up to a $3 \mathrm{~cm}$ extent rostro-caudally in both SCI models, we consider that saline hypothermia (which might be beneficial, partially returning motor function in minipigs) was strictly connected with significant tissue preservation, at least within the immediate rostro-caudal (+1 and -1) segments. Local hypothermia applied following $8 \mathrm{~N}$ SCI for $5 \mathrm{~h}$ significantly enhanced the gray and white matter (dorsal, lateral and ventral columns) preservation near the lesion site (+1 and -1), and improved the preservation of neurofilaments in the lateral columns of the same segments. This experimental group achieved faster recovery of hind-limb function and the ability to walk from one to three steps with consistent plantar-hoof stepping at nine weeks in comparison with non-treated animals. It seems that the preservation of axons in the lateral funiculi in the +1 and -1 segments (improvement by 25 and $19 \%$ respectively) and more rostrally or caudally, might be essential for favorable neurological outcome.

Axonal injury dispersion in the spinal cord includes mainly demyelination, loss of neurofilaments and failure of axolemma permeability. Tomko et al (36) reported that the lesion size after dorsal column lesion in rats expanded dramatically within the first two days, when its size reached the maximum $(40.2 \pm 12.1 \mathrm{~mm})$. Decline in neurofilament numbers was detected in the dorsal and ventrolateral white matter funiculi at the lesion epicenter and $5 \mathrm{~mm}$ in rostral and caudal directions of injury diffusivity after spinal compression injury in rats (37). As shown previously, the fundamental role of neurofilaments is to preserve axonal caliber, so its breakdown represents deficiency of axonal conduction velocity $(38,39)$. Schumacher et al (40) reported an association between neurofilament loss and hind-limb motor function deficiency after 

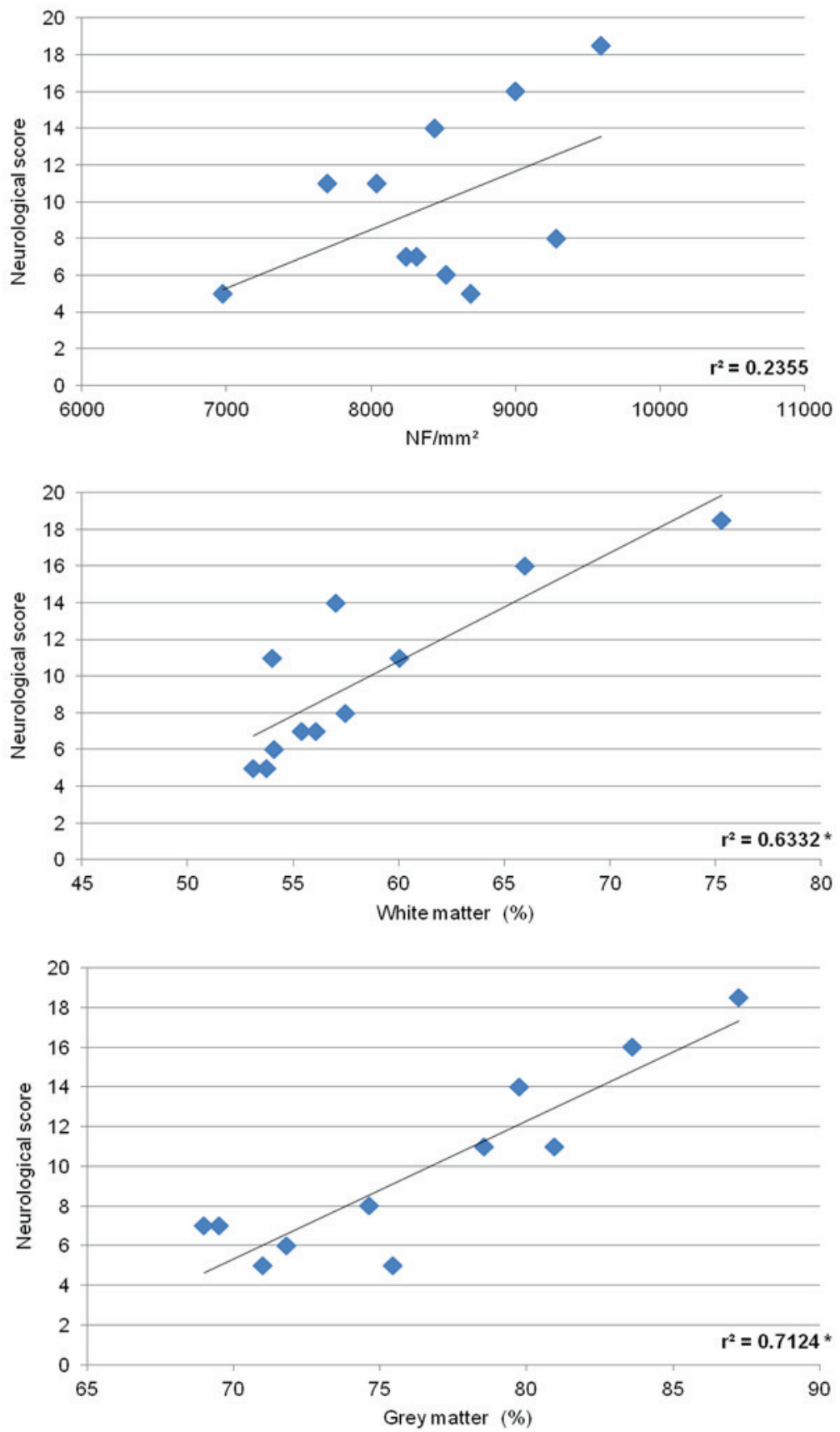

Figure 14. Correlation coefficients $\left(\mathrm{r}^{2}\right)$ between the degree of neurological recovery and neurofilament immunoreactivity, and white and gray matter preservation measured nine weeks after spinal cord injury ( $8 \mathrm{~N}$ and $15 \mathrm{~N}$ impact) and hypothermia with saline. There was a high degree of correlation with significance in gray and white matter preservation. Data are given as means $\pm \operatorname{SEM}(n=11) ;{ }^{*} \mathrm{P}<0.05$ with respect to neurological outcome.

SCI. Recently, Navarro et al (15) detected a loss of NF-positive axons four and nine months after Th12 contusion in minipigs in areas heavily infiltrated with IBA-1 positive cells. Nine weeks after SCI we observed significant reduction in the number of neurofilaments. Major increase in NF-IR profiles was detected after saline hypothermia in LC in the +1 and -1 segments, a finding which strongly correlates with significant white matter preservation. Our findings confirm the strong correlation between neurological outcome, the extent of tissue preservation within the immediate rostro-caudal ( +1 and -1$)$ segments, and preservation of neurofilaments which are almost exclusively found in myelinated axons. Such improvements were not observed in the saline-treated animals subjected to more severe (15N) SCI or in the group treated with DMEM/F12 medium. DMEM/F12 perfusion medium was designed to support the growth and maintenance of a variety of cells. The lack of its positive effect may have been due to the use of this medium at low temperature, which reduces its efficiency.

Saline solution administered over the lesion site in the $15 \mathrm{~N}$ SCI group significantly preserved the gray and white matter and protected neurofilaments in the dorsal and lateral columns, however the enhancement of tissue integrity was seen only in caudal segments. Devaux et al (41) reported that segments caudal to the lesion site hosted a robust inflammatory process, accompanied at least temporarily by local synthesis of neuroprotective and regenerative molecules. Most experimental 

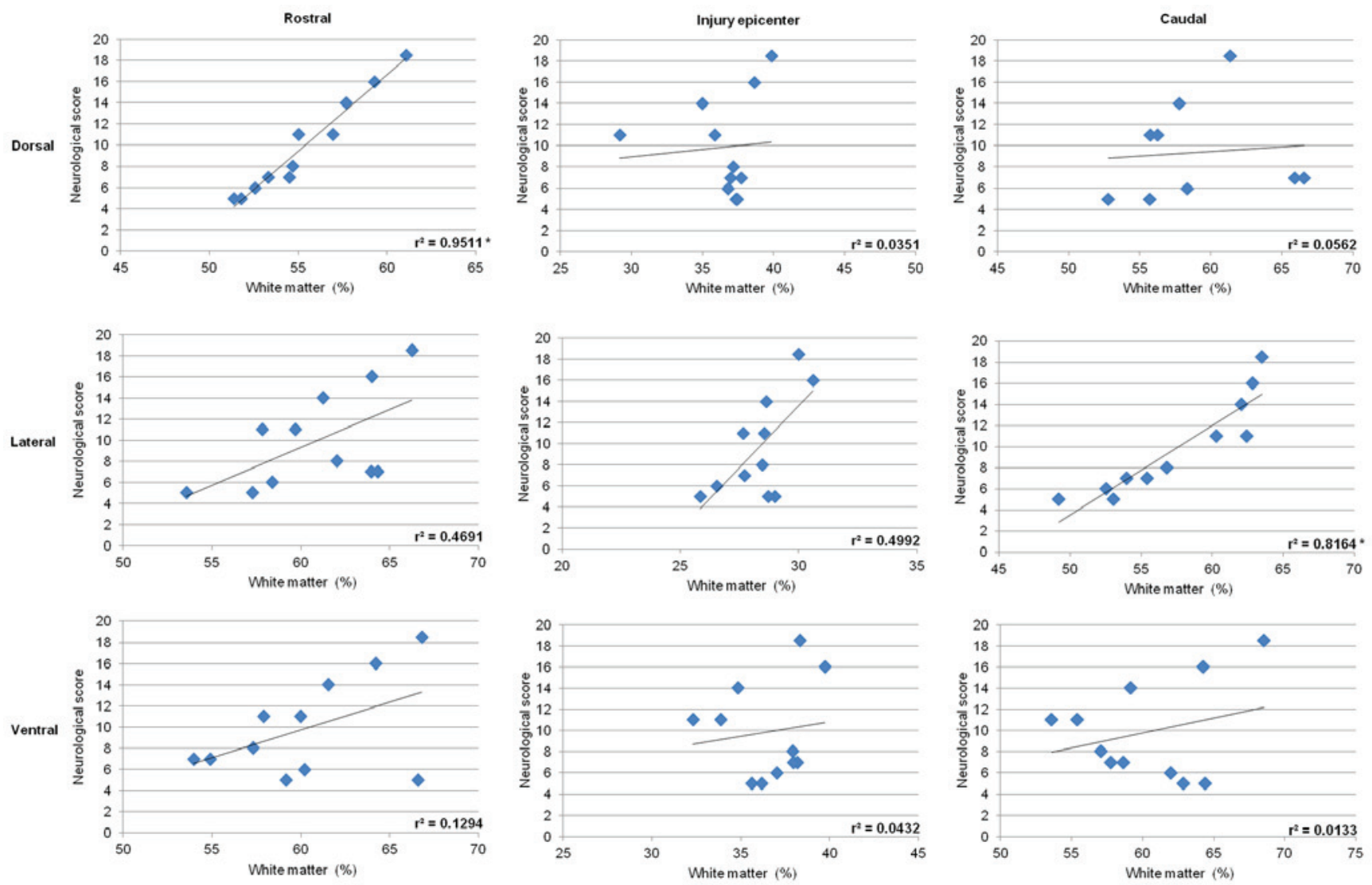

Figure 15. Plotted values show correlation coefficients $\left(\mathrm{r}^{2}\right)$ between neurological score and white matter preservation analyzed in the dorsal, lateral and ventral columns nine weeks after spinal cord injury ( $8 \mathrm{~N}$ and $15 \mathrm{~N}$ force impact) and hypothermia with saline. Spinal cord was taken from the lesion site, and rostral and caudal sections. Data are given as means \pm SEM ( $n=11)$; ${ }^{*} \mathrm{P}<0.05$ neurological outcome with respect to white matter preservation.
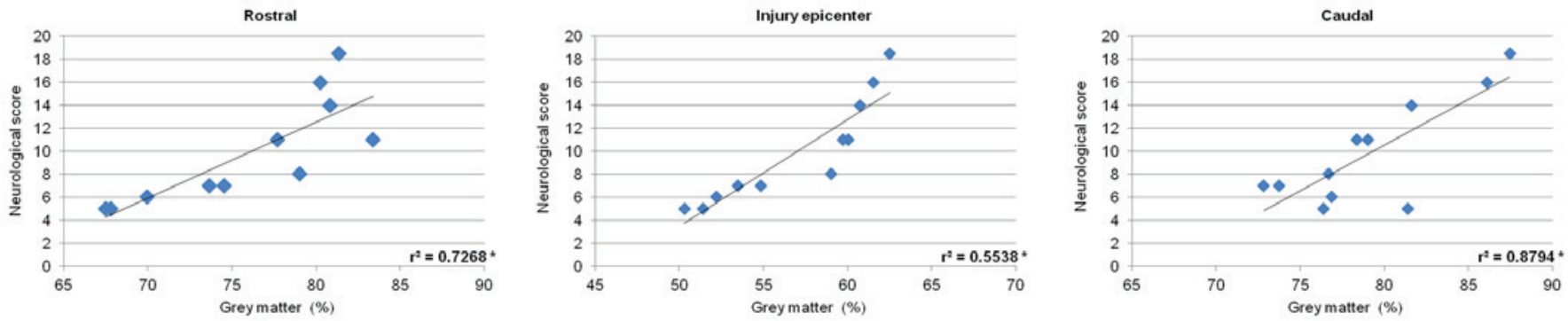

Figure 16. Correlation between locomotor function recovery and gray matter preservation nine weeks after spinal cord injury ( $8 \mathrm{~N}$ and $15 \mathrm{~N}$ impact force) and hypothermia with saline. There were high correlation coefficients $\left(\mathrm{r}^{2}\right)$ with significance between behavioral score and preserved gray matter in each spinal cord section. Data are given as means \pm SEM $(n=11) ;{ }^{*} \mathrm{P}<0.05$ neurological outcome with respect to gray matter preservation.

SCI studies show proliferation and activation of microglia in the injured spinal cord $(42,43)$. This proliferation accelerates neuronal damage by releasing toxic substances, including nitric oxide, super oxide and tumor necrosis factor-alpha $(\mathrm{TNF}-\alpha)$ (44-46). Our previous study revealed strong increase in NOS activity in the caudal segments immediately adjacent to the lesion site one day after mid-thoracic spinal cord constriction in rabbits (47). Morino et al (48) reported that peak proliferation of microglia in rats was around $48 \mathrm{~h}$, and the subsequent production of cytokine and nitric oxide were key events in accelerating neuronal damage after spinal cord injury. Mild hypothermia for $48 \mathrm{~h}$ inhibited microglia proliferation and TNF- $\alpha$ production after traumatic SCI and improved hind-limb motor function (49). In another computer-controlled minipig SCI model, the presence of high-density activated microglia and macrophages was identified up to nine months after SCI (15). This indicates that if the target of hypothermia treatment is inflammatory response, the hypothermia treatment in more severe SCI models should be continued for a longer time after the SCI.

In the present study, we validated the effectiveness of an implantable spinal perfusion chamber (using 3D scanner technology) in a computer-controlled spinal cord trauma minipig model. The perfusion chamber could be successfully used for application of selective spinal cord hypothermia in large pre-clinical animal SCI models, aimed at maximum functional benefit as defined by recovery of motor function. Although the superfusion of cold saline was restricted to a 
relatively small spinal cord region (just above the lesion site), the effectiveness of treatment spread up to $3 \mathrm{~cm}$ both cranially and caudally. The treatment showed that tissue integrity in both cranial and caudal segments immediately adjacent to the lesion (+1 and -1) and substantial preservation of neurofilaments in the lateral funiculi of the same segments is needed for a better neurological outcome. No improvement of functional recovery was seen when treatment affected the tissue integrity only in the caudal segments. These results suggest that local saline hypothermia $\left(4^{\circ} \mathrm{C}\right)$ applied through a spinal perfusion chamber for $5 \mathrm{~h}$ could be a promising therapeutic method when combined with clinically-proven surgical (decompression) and medical treatments for SCI. However, further experimental studies are required to evaluate hypothermia treatment for its possible utility in human patients with SCI.

\section{Acknowledgements}

This study was supported by the project: Formation and development of a diagnostic procedure in the treatment of trauma-injured spinal cord (ITMS 26220220127), supported by the Research and Development Operational Programme funded by the ERDF. We are grateful to S. Gancarcikova, MVD., PhD. for preparation of protocols approved by the State Veterinary and Food Administration and Mrs. A. Kosova for her technical assistance.

\section{References}

1. Arrica M and Bissonnette B: Therapeutic hypothermia. Semin Cardiothorac Vasc Anesth 11: 6-15, 2007.

2. Erecinska M, Thoresen M and Silver IA: Effects of hypothermia on energy metabolism in mammalian central nervous system. J Cereb Blood Flow Metab 23: 513-530, 2003.

3. Westergren H, Farooque M, Olsson Y and Holtz A: Motor function changes in the rat following severe spinal cord injury. Dose treatment with moderate systemic hypothermia improve functional outcome. Acta Neurochir (Wien) 142: 567-573, 2000.

4. Westergren H, Farooque M, Olsson Y and Holtz A: Spinal cord blood flow changes following systemic hypothermia and spinal cord compression injury: An experimental study in rat using laser-Doppler flowmetry. Spinal Cord 39: 74-84, 2001

5. Chatzipanteli K, Yanagawa Y, Marcillo AE, Kraydieh S, Yezierski RP and Dietrich WD: Posstraumatic hypothermia reduced polymorphonuclear leukocyte accumulation following spinal cord injury in rats. J Neurotrauma 17: 321-332, 2000.

6. Lo TP, Cho KS, Garg MS, Lynch MP, Marcillo AE, Koivisto DL, Stagg M, Abril RM, Patel S, Dietrich WD and Pearse DD Systemic hypothermia improves histological and functional outcome after cervical spinal cord contusion in rats. J Comp Neurol 514: 433-448, 2009.

7. Morino T, Ogata T, Takeba J and Yamamoto H: Microglia inhibition is a target of mild hypothermic treatment after the spinal cord injury. Spinal Cord 46: 425-431, 2008

8. Yu CG, Jimenez O, Marcillo AE, Weider B, Bangerter K, Dietrich WD, Castro S and Yezierski RP: Beneficial effects of modest systemic hypothermia on locomotor function and histopathological damage following contusion-induced apinal cord injury in rats. J Neurosurg 93(1 Suppl): S85-S93, 2000 .

9. Kida Y, Takano H, Kitagawa $\mathrm{H}$ and Tsuji $\mathrm{H}$ : Effects of systemic or spinal cord cooling on conductive spinal evoked potentials Spine (Phila Pa 1976) 19: 341-345, 1994.

10. Marsala M, Galik J, Ishikawa T and Yaksh TL: Technique of selective spinal cord cooling in rat: Methodology and application. J Neurosci Methods 74: 97-106, 1997.

11. Inamasu J and Ichikizaki K: Mild hypothermia in neurologic emergency: An update. Ann Emerg Med 40: 220-230, 2002.
12. Shibuya S, Miyamoto O, Janjua NA, Itano T, Mori S and Norimatsu H: Post-traumatic moderate systemic hypothermia reduces TUNEL positive cells following spinal cord injury in rat. Spinal Cord 42: 29-34, 2004

13. Dath R, Ebinesan AD, Porter KM and Miles AW: Anatomical measurements of porcine lumbar vertebrae. Clin Biomech (Bristol, Avon) 22: 607-613, 2007.

14. Rydevik BL, Pedowitz RA, Hargens AR, Swenson MR, Myers RR and Garfin SR: Effects of acute, graded compression on spinal nerve root function and structure: An experimental study of the pig cauda equina. Spine (Phila Pa 1976) 16: 487-493, 1991.

15. Navarro R, Juhas S, Keshavarzi S, Juhasova J, Motlik J, Johe K, Marsala S, Scadeng M, Lazar P, Tomori Z, et al: Chronic spinal compression model in minipigs: A systematic behavioral, quanlitative and quantitative neuropathological study. J Neurotrauma 29: 499-513, 2012.

16. Bernards CM: Understanding the physiology and pharmacology of epidural and intrathecal opioids. Best Pract Res Clin Anaesthesiol 16: 489-505, 2002.

17. Clément R, Malinovsky JM, Hildgen P, Dollo G, Estèbe JP Chevanne F and Le Verge R, LeCorre P: Spinal disposition and meningeal permeability of local anesthetics. Pharm Res. 21: 706-716, 2004

18. Durant PA and Yaksh TL: Distribution in cerebrospinal fluid, blood and lymph of epidurally injected morphine and inulin in dogs. Anesth Analg. 65: 583-592, 1986

19. Sulla I, Boldizar M, Racekova E and Balik V: Thoracic laminectomy technique in minipigs. Folia Veterinaria 56: 35-39, 2012.

20. Lee JH, Jones CF, Okon EB, Anderson L, Tigchelaar S, Kooner P, Godbey T, Chua B, Gray G, Hildebrandt R, et al: A novel porcine model of traumatic thoracic spinal cord injury. J Neurotrauma 30: 142-159, 2013.

21. Marsala M, Vanicky I, Galik J, Radonak J, Kundrat I and Marsala J: Panmyelic epidural cooling protects against ischemic spinal cord damage. J Surg Res 55: 21-31, 1993.

22. Vanicky I, Marsala M, Gálik J and Marsala J: Epidural perfusion cooling protection against protracted spinal cord ischemia in rabbits. J Neurosurg 79: 736-741, 1993.

23. Grulova I, Slovinska L, Nagyova M, Cizek M and Cizkova D: The effect of hypothermia on sensory-motor function and tissue sparing after spinal cord injury. Spine J 13: 1881-1891, 2013.

24. Levi AD, Green BA, Wang MY, Dietrich WD, Brindle T, Vanni S, Casella G, Elhammady G and Jagid J: Clinical application of modest hypothermia after spinal cord injury. J Neurotrauma 26: 407-415, 2009

25. Hansebout RR and Hansebout CR: Local cooling for traumatic spinal cord injury: outcomes in 20 patients and review of the literature. J Neurosurg Spine 20: 550-561, 2014.

26. Wang $\mathrm{J}$ and Pearse DD: Therapeutic hypothermia in spinal cord injury: The status of its use and open questions. Int J Mol Sci 16: 16848-16879, 2015.

27. Tetzlaff W, Kobayashi NR, Giehl KM, Tsui BJ, Cassar SL and Bedard AM: Response of rubrospinal and corticospinal neurons to injury and neurotrophins. Prog Brain Res 103: 271-286, 1994.

28. Yoshitake A, Mori A, Shimizu H, Ueda T, Kabei N, Hachiya T, Okano $\mathrm{H}$ and Yozu R: Use of an epidural cooling catheter with a closed countercurrent lumen to protect against ischemic spinal cord injury in pigs. J Thorac Cardiovasc Surg 134: 1220-1226, 2007.

29. Eidelberg E, Straehley D, Erspamer R and Watkins CJ: Relationship between residual hindlimb-assisted locomotion and surviving axons after incomplete spinal cord injuries. Exp Neurol 56: 312-322, 1977.

30. Fehlings $\mathrm{MG}$ and Tator $\mathrm{CH}$ : The relationships among the severity of spinal cord injury, residual neurological function, axon counts and counts of retrogradely labeled neurons after experimental spinal cord injury. Exp Neurol 132: 220-228, 1995.

31. Basso DM, Beattie MS and Bresnahan JC: Graded histological and locomotor outcomes after spinal cord contusion using the NYU weight-drop device versus transection. Exp Neurol 139: 244-256, 1996.

32. Dimar JR II, Shields CB, Zhang YP, Burke DA, Raque GH and Glassman SD: The role of directly applied hypothermia in spinal cord injury. Spine (Phila Pa 1976) 25: 2294-2302, 2000.

33. Nout YS, Ferguson AR, Strand SC, Moseanko R, Hawbecker S, Zdunowski S, Nielson JL, Roy RR, Zhong H, Rosenzweig ES, et al: Methods for functional assessment after C7 spinal cord hemisection in the rhesus monkey. Neurorehabil Neural Repair 26: 556-569, 2012.

34. Fawcett J: Repair of spinal cord injuries: Where are we, where are we going? Spinal Cord 40: 615-623, 2002. 
35. Sudo H, Taneichi $\mathrm{H}$ and Kaneda K: Secondary medulla oblongata involvement following middle cervical spinal cord injury associated with latent traumatic instability in a patient with ossification of the posterior longitudinal ligament. Spinal Cord 44: 126-129, 2006.

36. Tomko P, Farkaš D, Č́ižková D and Vanický I: Longitudinal enlargement of the lesion after spinal cord injury in the rat: A consequence of malignant edema? Spinal Cord 55: 255-263, 2017.

37. Ward RE, Huang W, Kostusiak M, Pallier PN, Michael-Titus AT and Priestley JV: A characterization of white matter pathology following spinal cord compression injury in the rat. Neuroscience 260: 227-239, 2014

38. Sakaguchi T, Okada M, Kitamura T and Kawasaki K: Reduced diameter and conduction velocity of myelinated fibers in the sciatic nerve of a neurofilament-deficient mutant quail. Neurosci Lett 153: 65-68, 1993.

39. Liu Q, Xie F, Siedlak SL, Nunomura A, Honda K, Moreira PI, Zhua X, Smith MA and Perry G: Neurofilament proteins in neurodegenerative diseases. Cell Mol Life Sci 61: 3057-3075, 2004.

40. Schumacher PA, Siman RG and Fehlings MG: Pretreatment with calpain inhibitor CEP-4143 inhibits calpain I activation and cytoskeletal degradation, improves neurological function, and enhances axonal survival after traumatic spinal cord injury. J Neurochem 74: 1646-1655, 2000

41. Devaux S, Cizkova D, Quanico J, Franck J, Nataf S, Pays L, Hauberg-Lotte L, Maass P, Kobarg JH, Kobeissy F, et al: Proteomic analysis of the spatio-temporal based molecular kinetics of acute spinal cord injury identifies a time- and segment-specific window for effective tissue repair. Mol Cell Proteomics 15: 2641-2670, 2016

42. Watanabe T, Yamamoto T, Abe Y, Saito N, Kumagai T and Kayama H: Differential activation of microglia after experimental spinal cord injury. J Neurotrauma 16: 255-265, 1999.

43. Saganová K, Burda J, Orendácová J, Cizkova D and Vanický I: Fluoro-Jade B staining following zymosan microinjection into the spinal cord white matter. Cell Mol Neurobiol 26: 1463-1473, 2006.
44. Tanaka M, Sotomatsu A, Yoshida T, Hirai S and Nishida A: Detection of superoxide production by activated microglia using a sensitive and specific chemiluminescence assay and microglia-mediated PC12 h cell death. J Neurochem 63: 266-270, 1994.

45. Lukácová N, Halát G, Chavko $M$ and Marsala J Ischemia-reperfusion injury in the spinal cord of rabbits strongly enhances lipid peroxidation and modifies phospholipid profiles. Neurochem Res 21: 869-873, 1996.

46. Lukácová N, Kolesárová M, Kuchárová K, Pavel J, Kolesar D, Radonak J, Marsala M, Chalimoniuk M, Langfort J and Marsala J: The effect of a spinal cord hemisection on changes in nitric oxide synthase pools in the site of injury and in regions located far away from the injured site. Cell Mol Neurobiol 26: $1367-1385,2006$

47. Lukácová N, Cízková D, Marsala M, Pavel J, Jalc P, Sulla I, Kafka J and Marsala J: Effect of midthoracic spinal cord constriction on catalytic nitric oxide synthase activity in the white matter columns of rabbit. Neurochem Res 25: 1139-1148, 2000.

48. Morino T, Ogata T, Horiuchi H, Takeba J, Okumura H, Miyazaki T and Yamamoto H: Delayed neuronal damage related to microglia proliferation after mild spinal cord compression injury. Neurosci Res 46: 309-318, 2003

49. Morizane K, Ogata T, Morino T, Horiuchi H, Yamaoka G, Hino $M$ and Miura $\mathrm{H}$ : A novel thermoelectric cooling device using Peltier modules for inducing local hypothermia of the spinal cord: The effect of local electrically controlled cooling for the treatment of spinal cord injuries in conscious rats. Neurosci Res 72: 279-282, 2012.

(7) $\Theta$ This work is licensed under a Creative Commons Attribution-NonCommercial-NoDerivatives 4.0 International (CC BY-NC-ND 4.0) License. 